\title{
Visually Augmented Audio-Tactile Graphics for Visually Impaired People
}

\author{
T. GÖTZELMANN, Nuremberg Institute of Technology
}

Tactile graphics play an essential role in knowledge transfer for blind people. The tactile exploration of these graphics is often challenging because of the cognitive load caused by physiological constraints and their complexity. The coupling of physical tactile graphics with electronic devices offers to support the tactile exploration by auditory feedback. Often, these systems have strict constraints regarding their mobility or the process of coupling both components. Additionally, visually impaired people cannot appropriately benefit from their residual vision. This article presents a concept for 3D printed tactile graphics, which offers to use audio-tactile graphics with usual smartphones or tablet-computers. By using capacitive markers, the coupling of the tactile graphics with the mobile device is simplified. These tactile graphics integrating these markers can be printed in one turn by off-the-shelf 3D printers without any post-processing and allows us to use multiple elevation levels for graphical elements. Based on the developed generic concept on visually augmented audio-tactile graphics, we presented a case study for maps. A prototypical implementation was tested by a user study with visually impaired people. All the participants were able to interact with the 3D printed tactile maps using a standard tablet computer. To study the effect of visual augmentation of graphical elements, we conducted another comprehensive user study. We tested multiple types of graphics and obtained evidence that visual augmentation may offer clear advantages for the exploration of tactile graphics. Even participants with a minor residual vision could solve the tasks with visual augmentation more quickly and accurately.

CCS Concepts: • Human-centered computing $\rightarrow$ Accessibility systems and tools; Touch screens; Auditory feedback; Ubiquitous and mobile devices; • Hardware $\rightarrow$ Tactile and hand-based interfaces;

Additional Key Words and Phrases: Tactile graphics, audio-tactile, visually impaired, blind, orientation, global, accessibility, tangible user interfaces, augmented, 3D printing, capacitive, touch screen, marker, capacitive sensing

\section{ACM Reference format:}

Timo Götzelmann. 2018. Visually Augmented Audio-Tactile Graphics for Visually Impaired People. ACM Trans. Access. Comput. 11, 2, Article 8 (June 2018), 31 pages.

https://doi.org/10.1145/3186894

\section{INTRODUCTION}

Manifold types of graphics are ubiquitous in our daily life. Just as for sighted people, they play an essential role in knowledge transfer for blind people. For example, tactile illustrations and graphs are used in the educational sector to communicate special knowledge and spatial contexts. Tactile

Author's address: T. Götzelmann, Nuremberg Institute of Technology, Keßlerplatz 12, D-90489 Nuremberg, Germany; email: Timo.Goetzelmann@ohm-university.eu.

Permission to make digital or hard copies of all or part of this work for personal or classroom use is granted without fee provided that copies are not made or distributed for profit or commercial advantage and that copies bear this notice and the full citation on the first page. Copyrights for components of this work owned by others than ACM must be honored. Abstracting with credit is permitted. To copy otherwise, or republish, to post on servers or to redistribute to lists, requires prior specific permission and/or a fee. Request permissions from permissions@acm.org.

(C) 2018 ACM 1936-7228/2018/06-ART8 $\$ 15.00$

https://doi.org/10.1145/3186894 
maps, as an example of a comparatively complex kind of graphics, are used to support orientation and navigation of blind and visually impaired people. They can improve their spatial understanding in unfamiliar urban environments [9]. Many of these tactile graphics are mainly designed for blind people. According to estimations of the World Health Organization (WHO), globally there are about 39 million blind people, but about 246 million people have low vision [55]. In this article, we will use the term visually impaired for both groups. A majority of these people live in low-income settings, and in some countries, visually impaired persons do not get financial support from the government (such as blind persons do).

Ideally, any residual vision of visually impaired people could be used to take particular benefit from tactile graphics, and these should be affordable to this target group.

There are several ways to produce tactile graphics. Their physical representations can be produced by multiple printing technologies (e.g., References [47, 51]). Braille embossers can be used to print graphics by punching series of dots into a special thicker paper. Modern embossers are capable of higher printing resolutions and approximate lines and elevated areas by numerous adjoined dots. Microcapsule (swell) paper is a chemically processed paper that can be printed with monochrome drawings of graphics by ordinary printers where the dark parts swell when they are treated with controlled heat from a fuser. More recently, tactile graphics are being produced by 3D printers, which print elevated 3D models generated from various data sources (e.g., maps). This technology has several advantages that can be used for structuring graphical content as well as the simultaneous use of multiple materials, which is leveraged in this article.

However, printed tactile graphics from all these technologies have one characteristic in common-they have to be kept simple. There are multiple reasons for excluding Braille annotations from tactile graphics. First, only a smaller proportion of blind people are able to read Braille at all [4]. It can be assumed that an even smaller proportion of visually impaired people can read Braille labeled graphics. Another argument to exclude Braille labels is to limit the graphics' tactile complexity. As pointed out by Tatham [45], the extensive use of Braille annotations in tactile graphics (such as maps) may worsen the overall legibility. Additionally, similar to visual graphics, the alternative of using legends and keys may complicate the interpretation of tactile images due to reduced immediacy [18].

An alternative is the combination of tactile graphics with an interactive component. Recent studies [5] for tactile maps have shown evidence that interactive audio support is advantageous in relation to the ISO 9241 usability design goals. There are numerous approaches for audio-tactile graphics. In this article, we present an interactive approach that can be used with existing tactile graphics but addresses, in addition to verbal descriptions, the visual modality of visually impaired persons. Because these tactile graphics can be interactively supported by both auditory and visual cues, we refer to them as Audiovisual Tactile Graphics.

This article is an expanded version of the original conference paper [11]. It emphasizes that the concept can be used for tactile graphics in general and illustrates its feasibility by a case study on tactile maps. Additionally, it reports from a second comprehensive user study that is measuring the effect of the visual augmentation incorporating several types of graphics. Compared to the first user study it includes more participants with a broader range of ages. This study is also reporting about detailed comments of visually impaired people and points out continuative issues.

\section{RELATED WORK}

Tactile graphics are available in many different forms. Beside the existing tactile graphics, visual graphics may serve as a basis to generate tactile representations. Multiple researchers proposed methods to automatically convert bitmap images into tactile graphics (e.g., References [10, 51]). 
Krufka and Barner [30] proposed a system to automatically produce tactile images from scalable vector graphics. Jayant et al. [22] investigated the process of generating tactile graphics and propose automated means to ease the production of tactile materials using image-based techniques. Li et al. [33] presented a system to translate photos into tactile graphics.

Other researches generate tactile graphics from other data types. Rotard and Ertl [34] generated tactile images from 3D objects by rendering line drawings and presented them on a tactile display. VizTouch [6] represented an approach to generate tactile data visualizations that were printable by 3D printers. Götzelmann and Pavkovic [14] presented an approach for generating 3D printable tactile maps accessing a map database that included buildings and blind-specific features. Finally, Kim and Yeh [27] presented a concept to generate 3D printable, movable tactile pictures for children with visual impairments using XML and CSS.

The presented techniques proposed techniques to generate tactile representations. Since the auditory augmentation of tactile graphics can be advantageous for the understanding of visually impaired users (e.g., Reference [5]), there are numerous approaches for audio-tactile graphics that use various techniques to support blind people. We report some of the most relevant approaches from a technological perspective toward a purpose-oriented manner in relation to our solution.

\subsection{Integrated Approaches}

These approaches directly integrate electronic representation with electronic components, i.e., do not have independent tactile representations. Some approaches mainly rely on communicating graphical elements using software and standard hardware built in off-the-shelf computers or mobile devices. Approaches relying on sonification encode tactile elements as sound (e.g., Timbremap [43]). Other approaches use touch inputs to explore electronic images by playing sound files when the user enters specific regions of the touch input (e.g., Reference [21]). Experiments show that this strategy may contribute to the spatial understanding of blind and visually impaired people. TouchOver Map [39] displayed street networks on mobile devices and used a vibration actuator and speech to guide blind users along displayed streets. Access lens [25] tracks finger movements on paper documents and provides audio feedback by using an OCR approach. Other approaches integrate devices for tactile feedback with electronic components and offer audio feedback. Iglesias et al. [20] introduced an approach based on the GRAB system to explore graphics such as 3D models and data charts by the sense of touch. It combined a dual-finger haptic interface with verbal explanations and voice input. Another haptic device, the Phantom, was used in several approaches for audio-tactile exploration (such as Reference [8]) of graphical information. Wall and Brewser [48] introduced a system for bimanual interaction. Whilst the dominant hand of visually impaired people was using a graphics tablet for input. The non-dominant hand received feedback by a specialized computer mouse VTPlayer, which integrated two tactile pin arrays. Audio output was used to provide information about the tactile representation to reduce cluttering. Petit et al. [38] used a refreshable tactile device $S T R e S S^{2}$, which facilitates piezo actuators to stretch the skin of the finger. They experimented with multiple types of scientific graphics such as diagrams, bar-charts, and maps from school books. In their comprehensive user study, blind people were supported by the system by audio feedback when they explored the graphics.

There are several works that utilize Hyperbraille, a technology mainly for blind people that uses the combination of a tactile display (a matrix of piezo actuators) with a desktop computer. There were multiple approaches from Zeng et al. (e.g., References [58-60]) for interactive tactile maps that adapted their tactile rendition due to a user's touch interaction. Another approach [40] addressed the tactile exploration of building plans and outdoor environments, which was supported by a text-to-speech (TTS) functionality. Adaptability is a key strength of this technology. Displayed graphics can adapt their tactile renditions dynamically and interactive augmentation techniques 
(such as blinking encoded by oscillating tactile elements) can be implemented. Another technology was Linespace [44], a combination of a camera and a modified 3D printer attached to a whiteboard. Graphical updates (such as zooming) could be initiated by people who are visually impaired whilst preserving parts of the previous rendition of the graphical contents. One main advantage compared to Hyperbraille was the significantly lower production costs, however its downside was the lack of immediacy. Both Linespace and tactile displays address a major claim by Jacobson [21], that updates of the tactile rendition cause a high cognitive load for visually impaired users if no context information is preserved. However, the drawbacks of these technologies are their limited mobility and availability.

\subsection{Approaches Augmenting Physical Graphics}

The following approaches use physical representations of tactile graphics in conjunction with electronic devices. The aim of these technologies is to provide additional information stored as electronic content to graphical elements the user is interested in. This helps to reduce the tactile complexity of graphics and, thus, addresses the issue of tactile cluttering particularly caused by textual annotations. The use of these two independent components makes it necessary to solve two main challenges of tangible user interfaces. First, the physical component has to be identified by the electronic system to couple both the electronic and physical entities. Second, to allow interaction with particular parts of the physical representation of the tactile graphic, its position and orientation have to be determined or restricted to specific constraints.

An early concept of audio-tactile graphics was the NOMAD board [37]. This system integrated a touch-sensitive surface with a computer. If the user touched parts of graphics mounted on the surface, then the system provided verbal explanations to the touch point on the graphics. A more flexible system was the Talking Tactile Tablet (TTT) [32], which combined a touch-sensitive surface and a desktop computer linked by a USB interface with tactile overlay sheets. When exchanging these sheets, they had a specified size and layout and had to be arranged exactly in the frame of the touch surface according to three calibration points. After justifying the sheets, the user had to touch multiple positions to register the graphics with the system. A graphical user interface was used to create graphics as source for the tactile sheets that are printable by local Braille embossers. A subsequent approach [36] allowed the automatic production of TTT sheets for U.S. maps optionally by third-party companies. The same company distributes a smart pen (TTPen), which augments special tactile sheets by audio explanations.

Wang et al. [49] described a system to analyze existing visual maps to initially transform them into a simplified image that can be printed by embossers or on microcapsule paper. These prints could be placed on a touch surface. During exploration of the tactile map, the automatically extracted metadata of street networks was used for verbal feedback to map features. Another approach, Touchplates [26], presented a system of a tabletop computer and acrylic plastic overlays combined with visual markers. It was able to recognize these overlays automatically by a technology called infrared based diffused illumination, which is integrated in tabletops such as Microsoft PixelSense. Brock et al. [5] used a system of a touch input space and raised-line drawings on microcapsule paper to examine multi-touch exploration of visually impaired people. The approach MapSense [7] extended this approach for a study focusing on visually impaired children, which incorporated 3D printed objects that could be placed on tactile maps.

These approaches allowed the exchangeability of tactile graphics and, partially, to recognize tactile graphics by performing a manual procedure of multiple steps. The necessity to place the map exactly in a certain way on the touch device and the restricted portability of these systems are the limitations of these approaches. 


\subsection{Mobile Approaches}

As a study of Espinosa et al. [9] showed, it may be useful that tactile graphics are portable. There were also several audio-tactile approaches that allow the transportation of both the electronic component and the tactile graphics. McGookin et al. [35] presented a study on the combination of opaque, raised paper overlays for non-visual interaction with dedicated applications using touch screens of mobile devices. When the participants interacted with the tactile overlays, sounds and verbal feedback were provided. Sennette et al. [41] presented a portable approach that allowed the use of tactile microcapsule prints of a popular cartographic database as an overlay for tablets and smartphones. After electronic map contents were loaded manually, the tactile maps were constrained to be placed exactly in line with the display's borders.

\subsection{Use of marker technologies}

Baker et al. [1] used manually attached visual markers (QR-Codes) encoding text in tactile graphics to assign verbal explanations to dedicated graphical elements. Standard smartphones were used to decode the marker the user was pointing at with his or her finger and to read aloud the corresponding explanation. They explored methods to capture the correct visual marker with the camera. Another portable approach [16] relied on a combination of smartphones and 3D printed tactile maps placed on a flat surface. Users were instructed by voice commands to hold the smartphone above the map to recognize it by inscribed visual markers (barcodes). An optical finger detection allowed users to query information about map features. A user study with blind users verified its feasibility, but this approach was considerably dependent on lighting conditions and constrained users to a one-handed exploration of the tactile map. An approach not focusing on tactile graphics (2.1/2.5D) but on 3D models [42] is using 3D printed acoustic markers attached on the 3D objects surface instead, which are strummed by the user to obtain dedicated verbal explanations. A smartphone is classifying the produced sounds and reads aloud associated texts.

The first approach, which combined tactile maps with markers that can be recognized by conventional smartphones and tablet computers, was CapMaps [12]. Hence, it addressed the issue of visual marker based approaches needing good lighting conditions and allowed two-handed exploration of the map. A succeeding approach [11] adapted this utilizing translucent (or semitransparent) materials and focused on the technical feasibility of such an approach. Based on this technical paper, this journal article will address tactile graphics in general. To evaluate the advantages of visual augmentation in tactile graphics, it reports from a second comprehensive user study about the effect of the visual augmentation for several types of graphics.

\subsection{Discussion}

Some of these approaches are portable. But even when the system supports interchangeable tactile graphics, users have to carry out a defined, multi-step procedure when changing a map. There are hard constraints for the arrangement of the graphics on the touch inputs that have to be met by blind people. Finally, none of the presented approaches investigates the additional use of visual augmentation for the exploration of audio-tactile graphics to support visually impaired people with residual vision. In the following, we introduce a novel approach to overcome these issues by the use of a novel type of marker technology and to additionally support visually impaired persons by augmentation of individual graphical features.

\section{APPROACH FOR VISUALLY AUGMENTED AUDIO-TACTILE GRAPHICS}

As pointed out in Section 1, our aim was to develop a mobile solution for audio-tactile graphics for both blind and visually impaired persons that uses auditory and visual augmentation. The 
development of our approach required several initial design considerations to solve general questions of technical design to make them usable with capacitive touch displays. Next, the step of exchanging tactile graphics, i.e., especially the effort required to mount the graphics on the touch display, had to be minimized. Finally, software-related questions for the detection of the physical tactile graphics had to be addressed.

\subsection{Technical Design Considerations}

Tactile graphics can be produced by multiple printing technologies. An inherent limitation of creating detailed tactile graphics using microcapsule paper is the support of only one elevation (height) level. Even worse, the elevation cannot be reliably controlled. Small features as well as thin lines may suffer from under-elevation, whilst extensive elevated areas are prone to heating artifacts when the fuser's temperature is set too high. Modern embossers are able to approximate height differences by varying the dots punched into the specialized paper.

3D printers are designed to fabricate spatial structures and thus can reproduce exact height differences. This technical feature can be exploited to support the grouping and differentiation of individual graphical elements, and thus the graphics readability. Another advantage is that 3D prints can be produced in a single pass whilst integrating multiple printing materials. This fact is leveraged in this paper to permit automatic recognition of graphics (see Section 3.2). However, currently available 3D printed tactile graphics cannot be used without modification for the joint use with touch displays. Multiple technical and physiological constraints have to be harmonized to adapt existing 3D printed tactile graphics.

Three main factors influence our design considerations: (i) the tactile acuity, i.e., the discriminability of the finger's skin, (ii) the effective resolution of consumer 3D printers, and (iii) the ability of capacitive inputs to recognize touches through a tactile graphic. When multiple elevation levels should be used in a tactile graphic, the latter factor imposes the requirement to minimize the height differences between elevation levels.

3.1.1 Resolution of Consumer 3D printers. There are 3D printers on the consumer market that work according to different printing techniques. The most common consumer 3D printers work according the Fused Decomposition Modelling (FDM) principle. Printers of this type use thermoplastic polymers, usually in the form of filament wound up on coils. By using stepper-motors for the $\mathrm{x}$ - and $\mathrm{y}$-axes, each point on the printing bed can be accessed. The printing head melts the filament and deposits it on points defined by the 3D model. This procedure is repeated layer per layer, whereas the printing bed (z-axis) moves away from the printing head(s) for a defined distance. By using printers with two or more printing heads, multiple printing materials can be used to fabricate (functional) multi-material 3D objects in a single turn.

The resolution of these printers is dependent on numerous factors. The most important characteristic for realizing multiple elevation levels for tactile graphics is the resolution on the z-axis (layer resolution). Current consumer FDM printers achieve up to $20 \mu \mathrm{m}$ layer resolution. However, printing with high resolutions may drastically increase printing time.

3.1.2 Discriminability of Elevation. Even fractions of a micron can be sensed due to deformations of the papillary ridges of the finger's skin [31]. However, this is only possible in specific settings, which are not applicable to 3D printed graphical elements. To ensure that the intended elevation of distinct graphical elements is not confused with 3D printing artifacts, at least the nominal layer resolution should be used.

Smaller elevations require more time for reading [24]. The optimal elevation with regard to scanning time between two elevation levels has been determined to start at $160 \mu \mathrm{m}$ [23]. According 

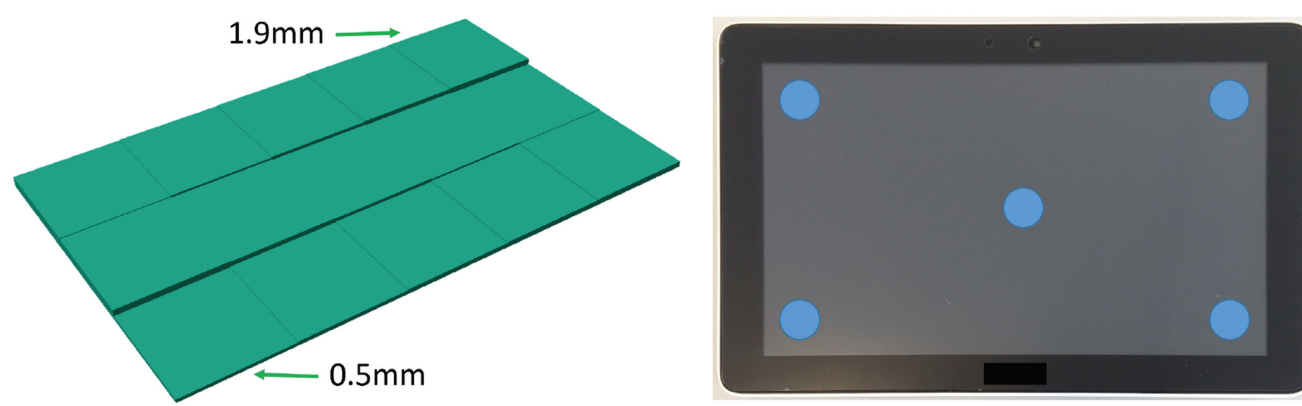

Fig. 1. Ramp of height differences to measure the range of distances for proximity touches of touch displays (at blue dots).

to Way and Barner [52], the exploration of tactile images follows two stages. First, the entire image is explored to gather a general overview, and second, detailed information of specific parts is obtained. We adapt this strategy to the elevation levels of 3D printed tactile graphics. On this basis, we propose that differences in elevation between graphical elements should be elevated at least $160 \mu \mathrm{m}$. Only if needed, fractions of this value should be used to allow the discrimination of differences between graphical elements in example by textures.

3.1.3 Sensing Abilities of Capacitive Touch Inputs. Current mobile devices usually integrate capacitive touch displays that enable the registration of at least 10 concurrent touches by the device's software. The user interactions are tracked by frequent determination of the individual fingers' positions, which touch the display's surface. To locate the touch positions, a sensitive microcontroller (e.g., Atmel maXTouch) senses load changes in a uniform grid of sensor wires attached to the touch display. This grid may be located directly on the surface (surface capacitance) or be placed below an insulating surface, such as robust glass (projected capacitance). The latter type is used for modern mobile devices. An important factor is that load changes in the sensor grid can already be detected when a finger approaches the touch surface. Hardware manufacturers adjust their touch microcontrollers well to maintain an equalized touch sensitiveness over the whole display area. This controlled sensitiveness allows it to sense touches through thin non-conducting materials (e.g., paper, plastics, glass). In this article, we make use of these technical characteristics and refer in the following discussion to such indirect touches as proximity touches.

To find good parameters for the generation of 3D printable tactile images using multiple elevation levels, an initial study is necessary to find out the range of distances where proximity touches are detected by touch displays.

Methodology. We evaluated discriminable height differences in current mobile devices. We tested 20 devices to estimate the range of proximity to the display. We tested it through a 3D printed PLA ramp with ascending height differences by utilizing the open-source constructive solid modelling tool OpenSCAD [28]. It consisted of multiple adjacent platforms of $1.5 \mathrm{~cm}^{2}$ and height differences of $0.1 \mathrm{~mm}$ in the range of $0.5-1.9 \mathrm{~mm}$ (see Figure 1). For each device, five measuring points (center and the display's corners) were evaluated. Each measuring point was touched a total of five times in decreasing distances from $1.9 \mathrm{~mm}$. We recorded the maximum distance proximity touches when each of the touches could be correctly recognized. 
Results. The range of medium distances between devices ${ }^{1}$ was $0.58-1.90 \mathrm{~mm}(\varnothing, 1.17 \mathrm{~mm} ; \sigma$, $0.40 \mathrm{~mm}$ ). Hence, each of the devices was able to detect proximity touches on average up to $0.58 \mathrm{~mm}$ distance. However, more than $75 \%$ of the tested devices were able to detect proximity touches of $\leq$ $0.99 \mathrm{~mm}$. According to the results of our preliminary study on touch proximity, $75 \%$ of the tested devices were not able to detect proximity touches from a distance above $1.43 \mathrm{~mm}$. This information is useful to define the minimum distance of conductive materials, which should not cause a proximity touch (i.e., the bridging component of the capacitive codes described in Section 3.2).

Discussion. Based on the results of this study, we argue that the elevation levels used for encoding tactile graphics' elements should remain below $1.0 \mathrm{~mm}$ to ensure that the interaction on the tactile graphics by proximity touches is possible. Combined with the physiological characteristics of tactile perception, this makes it possible to have 4-5 elevation levels. This additional level of freedom in can be used for tactile graphics design, to differentiate graphical elements or to implement a tactile perceivable hierarchy in the graphics.

\subsection{Recognition of Tactile Images}

Capacitive coupling of human fingers is sensed by touch surfaces. By using conductive materials, this coupling can be passed along the surface (e.g., Sketch-a-TUI [53]) or through objects (e.g., CapWidgets [29]).

When the capacitance of the user's finger is bifurcated to different locations on the touch surface, multiple concurrent touches are registered when the user touches this element [15]. The principle of bridging multiple points on the touch surface by conductive material can even be realized for untouched detection [46]. The concept of capacitive codes is based on the fact that distances between concurrent touch points can be measured. Because of this, these distances can be used to encode information.

We apply this principle to encode an identification of the tactile graphics ${ }^{2}$. Two touch points have at a minimum distance of more than $10 \mathrm{~mm}$ to be detected as individual points. Larger distances between two points can be measured with an accuracy of at least $5 \mathrm{~mm}$. Our images ID code consists of four points in a row. The first two points are at the minimum distance of $11 \mathrm{~mm}$ and mark the start of code (see Figure 2). A third point (Code A) is placed at least at the minimum distance $+5 \mathrm{~mm}$, to distinguish it from the beginning of the code. The fourth point (Code $B$ ) is placed in the same manner relatively to the third point. By varying the distances of Code A and B by multiples of $5 \mathrm{~mm}$, numerous combinations can be achieved. This code is integrated into the tactile images 3D model and printed using conductive filament.

When the interactive application is started on the mobile device, it first requests that the tactile graphic with such an integrated capacitive code is placed on the touch display and for the user to touch or move it. This causes the registration of four concurrent touch points by the interactive application. The application detects the start of code (which is always two points at the distance of $11 \mathrm{~mm}$ ) and then measures the distances to both of the other touch points to extract the ID code. Besides the ID code, the exact position and orientation of the tactile graphics is extracted from the registered touch points, which allows us to adapt the electronic content to this orientation. In

\footnotetext{
${ }^{1}$ Tested devices (Focus was on current lower cost tablets. Smartphones are marked italic): Acer Iconia One 10; Acer Predator 8; Apple iPad mini 4; Apple iPad Pro 12.9; Apple iPad Pro 9.7; Archos 101 Xenon Lite; Archos 7.0c Xenon; Asus Zenpad 10.1; Huawei MediaPad 10 Link + ; Lenovo Yoga Tablet 3; LG G Flex 2; Odys Score Plug 3D 10.1; Samsung Galaxy Tab E 3G; Samsung Galaxy Tab E Wifi; Samsung Galaxy Tab 7; Samsung Tab S 10.5; Samsung Tab S2 9.7; Trekstor Surftab Breeze 7.0 Quad 3G; Wiko Getaway; Xoro Telepad 7 A3.

${ }^{2}$ Parts of this section have been previously published in Ref. [12].
} 


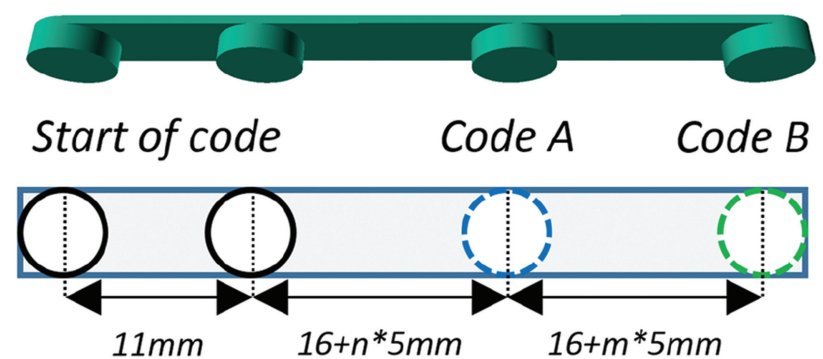

Fig. 2. The ID code that is embedded in the tactile graphic: varying distances by variables $\mathrm{n}$ and $\mathrm{m}$ encode a unique identifier that can be recognized through touch-displays.

particular, the users touch inputs on the tactile image can be accurately mapped to coordinates on the electronic representation of the graphics, even when the tactile image is shifted or rotated.

As with any other audio-tactile graphics application, has to assign location-dependent user interaction with the tactile graphics to commands. Dependent on the specific type of the tactile graphics the interactive application has to define regions of the tactile graphics, in which specific verbal explanations have to be provided and elements have to be visually augmented.

\section{FEASIBILITY STUDY BY A CASE STUDY ON MAPS}

As a proof-of-concept, we carried out a feasibility study including working prototypes of a common type of audio-tactile graphics, i.e., audio-tactile maps. These prototypes used the capacitive codes proposed in the last section and adapted the obtained technical parameters for designing the tactile elements. Multiple approaches exist to generate tactile maps and to automatically assign verbal explanations and visual augmentations to graphical elements of the map. These maps, called LucentMaps, were translucent to be visually augmented. For this study, we implemented a mobile application for detection of the tactile graphics, to provide verbal feedback and to visually augment parts of the graphics the user is interacting with.

\subsection{Interaction Concept}

First, we introduce our intended interaction concept. When a capacitive code is recognized, the actual user interaction starts.

4.1.1 General Requirements. The user is able to explore the tactile map, which is supported by touch interaction, speech input and output, and the visual augmentation of the map. The integration of capacitive codes into the printed map should minimize the effort required to interchange tactile maps. The software offers a simple way to initiate an exchange of the tactile map.

4.1.2 Touch Interaction. When exploring the map, users should have the opportunity to get immediate feedback about the map features they are interested in (such as its feature type and name). It is technically feasible to support many touch gestures through tactile maps. However, to not disturb the tactile exploration, we decided to keep the touch interaction simple. For the immediate feedback on specific map features, double tapping on the desired map feature was used. We followed the suggestions of Reference [5] to extend the time interval between the double tap to $700 \mathrm{~ms}$.

Since LucentMaps are designed to be used in an interactive context, we deliberately avoided integrating a scale into the tactile map. In contrast, the interactive application allows the 
Table 1. Elevation Levels and Their Associated Map Features

\begin{tabular}{|l|l|l|}
\hline Elevation & \multicolumn{1}{|c|}{ Map features } & \multicolumn{1}{c|}{ Examples } \\
\hline Level 1 & Superior areas & Building blocks, campuses \\
\hline Level 2 & Inferior areas & Buildings \\
\hline Level 3 & Walkable streets & Residential roads \\
\hline Level 4 & Footpaths & Pedestrian zones, walkways \\
\hline Level 5 & Points of Interest & Bus stops, shops, accessible crosswalks \\
\hline
\end{tabular}

The elevation level implements a hierarchy of features.

determination of arbitrary distance measurements by a multi-touch gesture. One finger has to mark the starting point of the measurement (e.g., a crossing) and a second finger marks the end point of the measurement by simply touching the desired end position (e.g., a corner of a building). The exact real-world distance is reported by the TTS. To not confuse blind or visually impaired users taking distance measurement with bimanual tactile exploration, this touch interaction has to be activated by an adequate voice command for each measurement.

4.1.3 Speech Interaction. To facilitate the tactile exploration of the map, the touch interaction is kept simple. To access additional functionality, speech input is used. To ensure acceptable response times and to be independent of internet access during exploration (e.g., when maps are used in situ), the speech detection works offline on the mobile device. The functionality covers (i) showing all map features of a certain class (point-, line-, area features), (ii) showing map features of specific categories (Health, Eating\&Drinking, Shops\&Services, Culture\&Entertainment, Nature\&Leisure, Transport\&Traveling, Accessibility) and should (iii) allow the user to obtain more detailed information about specific map features. Finally, the functionality mentioned before (measuring distances, locate and help user to be congruent to real-world directions, changing maps) should be accessible by speech input. The results of these interactions are also communicated by speech output. In the case of multiple map features, the TTS speaks the number of the augmented map features.

4.1.4 Visual Augmentation. The electronic map consists of area (e.g., buildings), line (e.g., streets) and point (e.g., traffic lights) map features. Visually impaired users should be able to augment each of these groups individually. Moreover, many of these map features are equipped with descriptive tags to characterize them (e.g., regarding their classification). Users should be able to augment each element of a specific class, (e.g., shops, pharmacies, public transport). Additionally, when selecting an area or line feature by double tap, the shape of this individual map feature should be augmented.

\subsection{Creation of Translucent Audio-tactile Maps}

There are several approaches for the automatic creation of 3D printable tactile graphics (see Section 2). For this article, we used a specialized web service (http://blindweb.org) to generate 3D models [14] usable for printing tactile maps. Its medium map detail level covers an area of $422 \times$ 287 meters; the actual map's scale is depending on its printed size (1:1875 for a printed size of $22.5 \times 15 \mathrm{~cm})$. We scaled the map height to the requirements of Section 3 and applied the following elevation levels for the map features (see Table 1).

By using its Python-API, we developed a plugin for the free open source 3D authoring tool Blender $3 D$ [2] to automatically adapt these maps to the constraints of our approach and to substitute them with capacitive codes. 

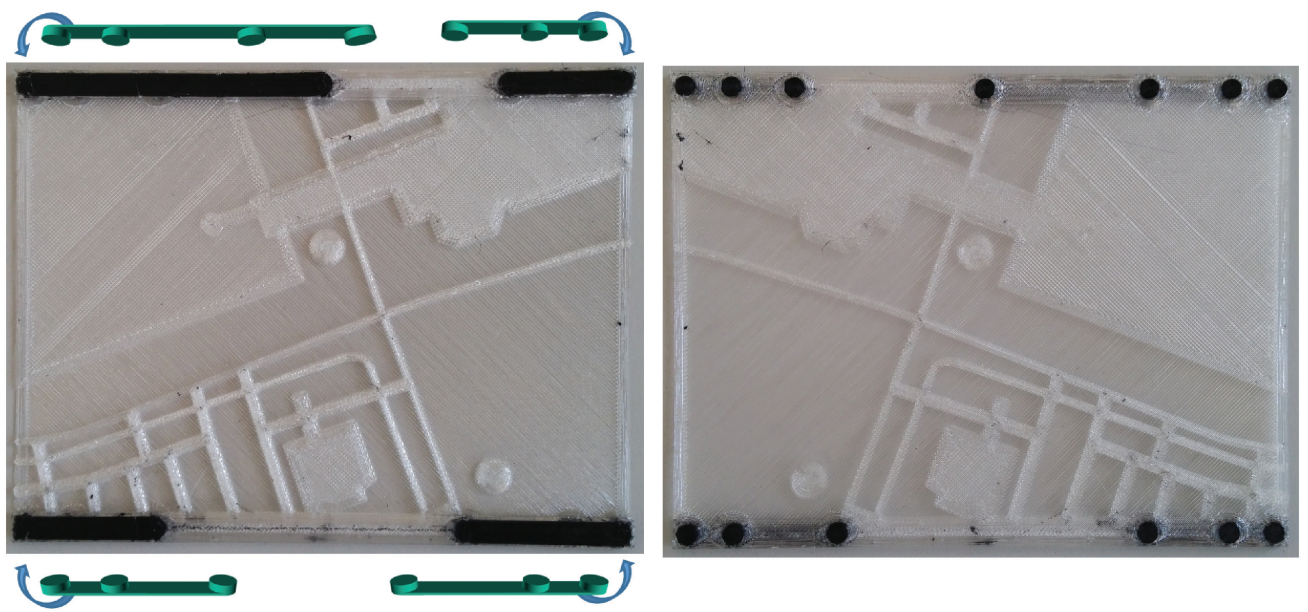

Fig. 3. Capacitive codes at tactile map's corners are printed with conductive filament. View from bottom side (right).
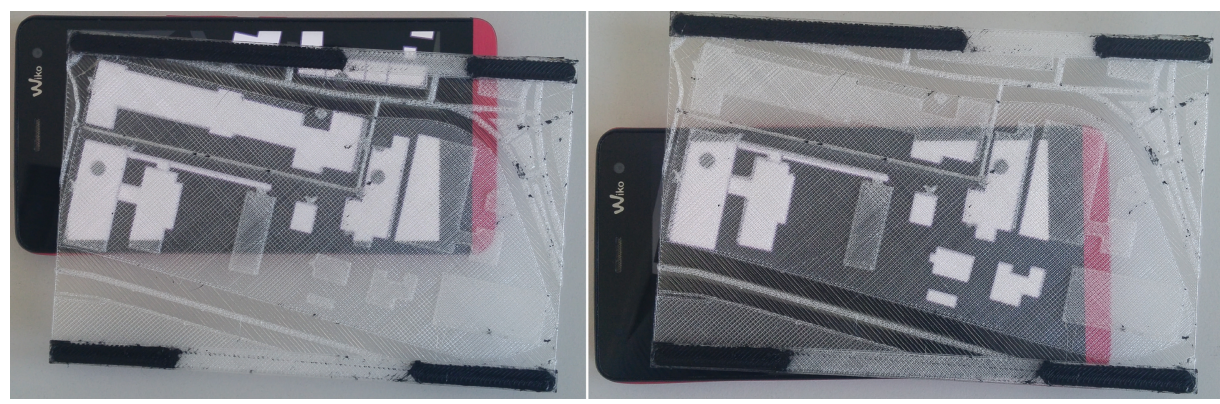

Fig. 4. User touches ID code at the map's top left corner and explores the map using a small device. By touching the code on the bottom left another part of the map can be explored.

For each individual map a dedicated ID was assigned. The LucentMaps' cardinal direction north was always on the top side. Since the ID code was located to the upper left of the tactile map (see Figure 3) this served as orientation for blind users who could feel the longish shape of the ID code. To make the maps also usable on small mobile devices, we integrated special codes for the remaining corners of the map. After the ID code of the map was recognized, these corner codes could be used to explore maps that are larger than the mobile device's display (see Figure 4). These corner codes only contain the start code and a third point, which encodes the corner ID by its distance to the start code analogous to Code A of the ID code.

The resulting multi-material 3D model was stored in two complementary 3D models (.STL), which share a joint coordinate system. This combination can be used by common 3D printing software for multi-material prints.

For the actual fabrication, we used an off-the-shelf dual-head 3D printer (FelixPrinters FelixPro 1), working according to the FDM principle. To print the map prototypes, we used a translucent filament, FormFutura HDglass, for the tactile map itself and Proto-pasta Conductive PLA filament for the integrated capacitive codes. The fewer layers used, the less light scattering occurs. Hence, 
to both minimize printing time and to maximize translucency of printed maps, we chose a layer resolution of $160 \mu \mathrm{m}$.

We intentionally omitted the integration of a wind rose or arrows for cardinal directions, because north on the maps is always represented by the upper bar containing the biggest capacitive code. On the one hand, this capacitive code can be used to find the cardinal directions relative to the map contents. On the other hand, the mobile device's compass could be used to obtain the orientation of the map contents relative to their real-world entities. Likewise, a fixed scale was not printed in the map, since the software allowed exact measurement of each distance the user is interested in by a two finger multi-touch gesture.

\subsection{Mobile Application}

We implemented a mobile application for Android tablet computers and smartphones that ran on at least API level 15 (Android 4.0.3), which was supported by the most current Android devices at the time of the study.

Implementation of functionality. For the recognition of the 3D printed tactile maps, we implemented an algorithm for detecting the capacitive codes embedded in the corners of the tactile map. When started, the application waits until it registers the four concurrent touch points in a line. Next, the detected touch points are classified. First, the start of code is extracted, followed by the classification of Code $A$ and $B$ based on their distance to the preceding point.

When the map's ID is determined, a dataset is retrieved including the geographic coordinates of the map's corners, its physical size and, optionally, a description of the map. Together with the computed coordinates and angle of the tactile map on the display, the electronic map is configured. The user is briefed about the recognized map.

For the map rendering and interaction, we relied on the Mapsforge $S D K{ }^{3}$ which has also been used for numerous other research works. It uses compressed maps from the OpenStreetMap database. Our application determined the geographic coordinates from touch interaction with the map even when the map view was rotated by the angle determined by the capacitive codes. For example, a double-tap on one position in the map queried the map database for features at that position. Then the information about the map feature's type and name was communicated by the TTS. The results of queries (e.g., to highlight all buildings) were visually augmented by adapting the rendering styles of the map.

For the speech interaction, we utilized the CMU PocketSphinx SDK [19] developed for offline speech detection mobile devices. It supports multiple detection models, such as context-dependent phonetic search, which allows good recognition results. Because of likely performance issues on some mobile devices, we had to use context-independent search that had to be tailored to our application to achieve reasonable results. To limit the unintentional triggering of voice commands, we defined the keyword "Computer," which is needed to start the actual recognition. We implemented a state machine to navigate through our auditory menu. To increase the detection accuracy, in each level only a small dictionary of recognizable words was used. The auditory menu structure by its detected words is listed below (curly brackets for submenu):

- Overview \{Buildings, Streets, Points\}

To show each representative of a feature class.

- Show \{Eating, Health, Travel, Shop, ... \}

To show representatives of a feature category.

$\overline{{ }^{3} \text { https://github.com/mapsforge/ }}$ 

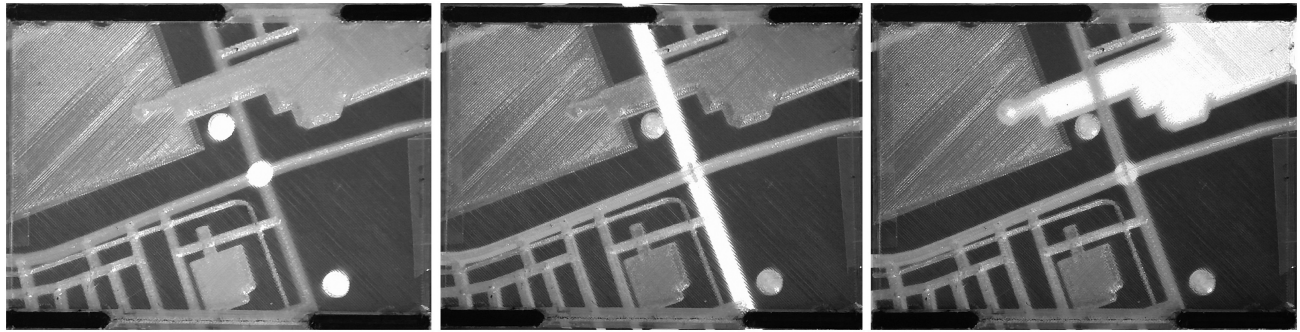

Fig. 5. User queries map for public transport and traffic map features (left), followed by a double-tap on the street (center), and finally on the building (right). For each of these interactions, the TTS tells name and type of the corresponding map features.

- Which \{Name, Type, Else\}

To obtain detailed information about a map feature the user points at.

- Map \{Locate, Distance, Change\}

To get information about GPS and orientation to real-world directions; to measure distances on the map; to change the tactile with another map (starting with recognition of capacitive code).

For text output, we used the standard Android TTS engine. For this study, we only used the normal voice speed. However, the speed can be easily set to user's preferences for optimal use.

\subsection{Usage Scenarios}

In the following, we define three intended usage scenarios to illustrate possible procedures of usage.

Blind person using tablet. Prior to a trip, the user wants to familiarize himself or herself with the target area. The user starts the application on a tablet computer and places an appropriate tactile map on its display. The user touches the capacitive code and waits for the acknowledgment that it has been recognized. Next, the user simply fixes the map by two strips of adhesive tape by holding the map on the display with one hand and attaching the strips by the other hand. Now, the user queries the map for public transport and street names to the destination, memorizes their shape, and measures distances. The user is also interested in descriptions of buildings surrounding these streets. This scenario could also apply to a visually impaired person (e.g., when searching for the way from a bus stop to a building), who is additionally supported by visual augmentation (see Figure 5).

Visually impaired person using smartphone. A visually impaired user wants to consult a tactile map on a journey after arriving at the target area by bus. He or she retrieves the smartphone and a map out of their pocket, places the map on the smartphone and touches the capacitive code. Subsequently, the TTS reports the description of the map and the map is fixed by adhesive tape. She or he initiates the location feature to orient the map to real-world directions and then explores a part of the map. Since the display is smaller than the map, another corner of the map is placed on the display and the corresponding corner code is touched. After the application recognizes the new corner of same map, it adapts and the map exploration continues.

Visually impaired person using tablet. The map is recognized just as in the previous scenarios. This time the user explores the map of a crowded area of a city center. By using voice commands, he 
or she gradually browses through the map feature categories and requests additional information about augmented map features of special interest.

\section{USER STUDIES}

\subsection{User Study on the Applicability of the Experimental Application}

To assess if blind and visually impaired people are able to use the approach proposed in Section 4 and to obtain qualitative feedback, we conducted a brief application-driven user study. Because we think the target group for interactive tactile maps are mostly younger people, we cooperated with the regional school for visually impaired and blind persons.

We recruited nine participants (five females) with an average age of $15.67 \pm 5.57$ years. Six of the participants were visually impaired (5-30\% residual vision), one had residual vision of $2 \%$ and two were blind. Each of the participants had basic knowledge about tactile maps. For the study, we used an inexpensive off-the-shelf Android tablet (Huawei MediaPad 10 Link+) with our implementation of the detection of capacitive markers and the interaction with the tactile map as described in Section 4.3. The display was set to maximum brightness, augmented parts of the map were represented by white color, whereas the remaining map features were indicated by a dark grey color.

Procedure. Initially the participants were given a brief description of the tactile maps and that mobile device touch displays are able to detect touches even through thin plastic. It was explained that capacitive markers are attached to the top and bottom sides of the map and that the tactile map can be automatically recognized by the tablet by touching or moving these markers on the tablet's display. Next, they had the chance to explore a trial map and were instructed to find the ID code, which is the largest (black) structure located on the upper left side of the map. Subsequently, multiple use-oriented tests were carried out with each of the participants.

1. The participants were asked to grasp the tactile map from the table and to place it on the tablet computer's display. Next, they had to locate and touch or move the ID code until they heard the TTS message that the map had been recognized. Finally, they had to shift and tilt the map to another position at least three times, using the capacitive code each time to reorient the system. After each shifting or tilting action they had to rest for a short while until the TTS finished reporting the changes in coordinates and angle.

2. After that, participants had to explore a tactile map fixed by adhesive tape on the tablet. They had to find each of a point-, line-, and area-feature and obtain verbal explanations by double tapping these map features. The visually impaired participants were asked to show the visually highlighted shapes of line- and area-features.

3. Next, voice commands were used to highlight

a. all restaurants,

b. all buildings, and

c. all point-features on the map. In each case, the visually impaired people were asked to determine the number of highlighted map features.

4. Finally, after activating the distance measuring feature by using the voice command, the participants were asked to measure the distance between two map features on the map by using a multi-touch gesture.

Results. All of the participants were able to place the tactile map on the tablet's display and to use the capacitive code for detection of the map. When the users relocated the map, this was detected by the application and the correct angle was reported (1). All of the participants were successful, none of the users needed numerous trials, which resulted in registration timings considerably less 


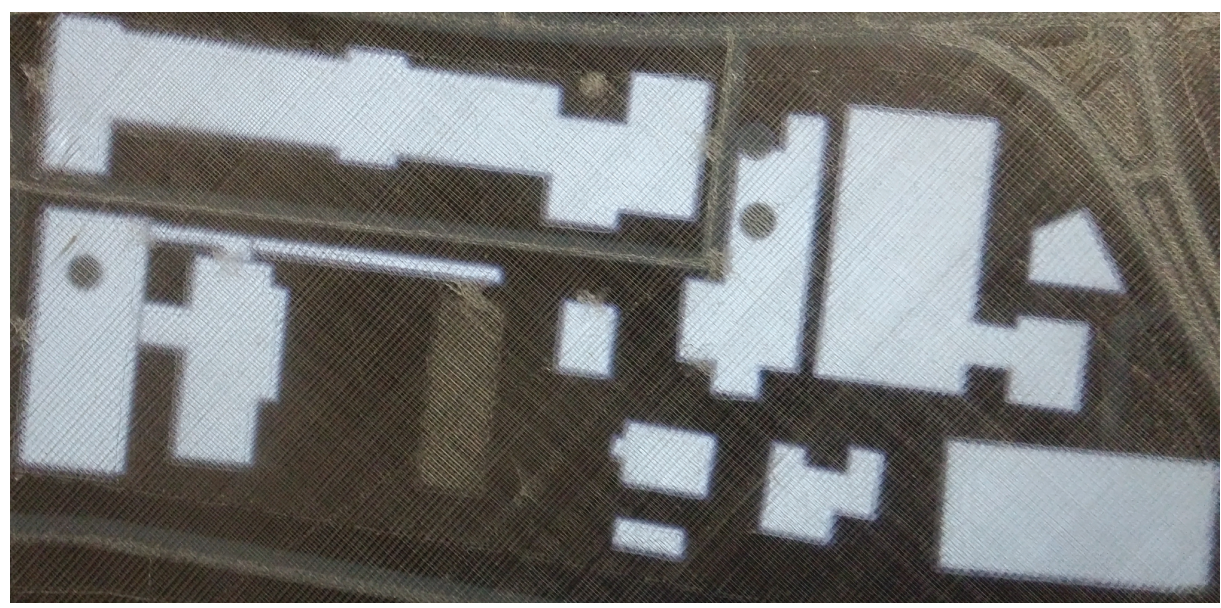

Fig. 6. Augmented tactile map: Adjacent buildings on the left were not recognized as individual buildings by one participant.

than 10s. Multiple users tried to rotate the map more than \pm 90 degree, which resulted in failure to recognize the code. However, this was a technical issue that could be solved by software in future versions.

All of the map users successfully managed the task to obtain additional information on point-, line- and area features (2). Visually impaired users recognized the rough shape of augmented map features and each of them recognized the correct number of augmented point features $(3 a+3 c)$. Four visually impaired participants recognized the correct number of 11 augmented buildings on the tactile map, one counted only 6 buildings and another was not able to see adjacent buildings (see Figure 6) as individual buildings. Each of the participants were successful to carry out the multi-touch gesture (4) to measure the distance between two map features.

Discussion. The aim of this user study was to test the technical feasibility of our use case on tactile maps. Both groups of people, blind and visually impaired, were curious to use the interactive approach. They obviously liked the fact that the map is automatically recognized when its capacitive code is touched on the tablet's display. Multiple users mentioned that it should be combined with a popular navigation application called BlindSquare, to be able to "feel" individual map features. One participant proposed to invert the augmentation of the map features, i.e., to augment the whole map except selected map features. Another participant liked the mobility of the map and the fact that small maps usable with smartphones or tablets do not attract attention of sighted people in contrast to large maps. Finally, the users had multiple suggestions on improvements of the map design. Even though map design is not the focus of this article, this information can be used for our future work.

Voice commands had to be repeated in some cases. We expect that outdoor use could be problematic and suggest using a Bluetooth headset for future applications. Despite the implemented keyword-search functionality, the voice recognition had to be disabled by the test supervisor between the individual tests, since sometimes unintentional commands were detected. For future versions, we suggest integration of a dedicated button in one of the display's corners, which would allow speech-recognition when it is pressed instead of the keyword-search functionality.

The results show the feasibility of our use case using the experimental application. It implemented the technical design considerations for visually augmented audio-tactile graphics (see 
Section 3.1) and used capacitive markers (see Section 3.2). None of the users had problems attaching the tactile overlays to the tablet computer, causing the system to recognize them by the markers, or to get verbal hints by touch interaction. Since the same components can be used for other types of audio-tactile graphics as well these technical results can be transferred to other application. The visually impaired people (including the person with $2 \%$ residual vision) were overwhelmed how much the visual augmentation of graphical elements can help. A comprehensive user study on the effect follows in Section 5.2.

\subsection{User Study on the Effect of Visual Augmentation of Tactile Maps}

The previous user study demonstrated the usability of the presented audio-tactile system for blind and visually impaired people. In this study particular attention was paid to the use of capacitive markers integrated with 3D printers with unmodified commercially available tablet computers. In particular due to the positive feedback of the visually impaired subjects, there were indications that the additional visual enrichment of the translucent audio-tactile materials can have an effect even with a high degree of visual impairment. This section reports on a more advanced user study that had the goal of investigating the effect of visual enrichment of tactile graphics.

5.2.1 Participants. We conducted the user study with 16 visually impaired people (eight female). Their ages were ranging from 19 to 49 years with an average of $34.63 \pm 5.24$. They were recruited from a vocational training center and a regional school for visually impaired and blind persons on a voluntary basis. No participants of the first study participated in this study. The sequence of tested participants was randomized.

Their reported visual acuity on the best eye ranged from $2 \%$ to $25 \%$, with an average of $6.69 \pm 9.82$. Two of them had a residual vision of $2 \%, 13$ between $2 \%$ and $10 \%$, and one participant had a residual vision of $25 \%$. Six participants were congenitally visually impaired, two more entered moderate visual impairment (at least by WHO category 1 [56]) with less than 18 years and the remaining participants later in their life. The causes for their visual impairments were as follows:

- Retinitis pigmentosa: 7 ,

- Cataract: 2,

- Spine-rod dystrophy: 2,

- Optic atrophy: 2,

- other diseases related to the optic nerve: 3 .

Most of the participants did not have $(n=6)$ or almost not have $(n=8)$ experience with tactile graphics. Only two of the participants stated that they had often used tactile graphics.

5.2.2 Apparatus. To ensure that the test results are not limited to a specific hardware platform, we intentionally did not use a high-end tablet computer for this user study. Instead, we used a 10-inch tablet computer Huawei MediaPad Link 10+, which was commercially launched in 2013 and costed less than $100 €$ when the study was carried out. Its display brightness $\left(\max .350 \mathrm{~cd} / \mathrm{m}^{2}\right)$ and the contrast ratio (max. 800:1) are weaker than those of many current tablet computers and thus, served well as a lower bound.

As in the feasibility study, all tactile elements were printed by 3D printing using the translucent filament $H D$ glass. Even though 3D printers are able to produce tactile graphics with several levels, only one level was used in this experiment. The basis for the printing height was the height of tactile-readable elements on microcapsular paper (i.e., Reference [50]). Therefore, the elements were printed with a total height of $0.3 \mathrm{~mm}$ consisting of two layers at a layer height of $0.15 \mathrm{~mm}$. 


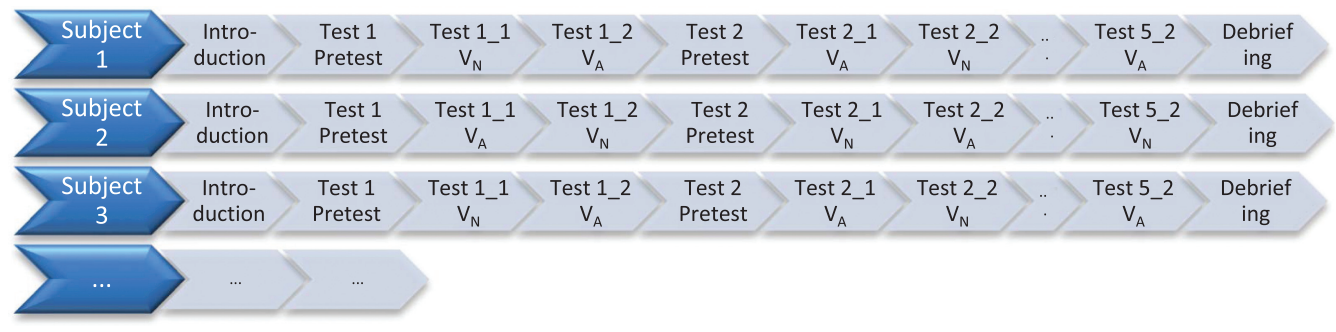

Fig. 7. Sequence of user study.

The 3D printed tactile examples were fixed on the tablet computer with two strips of translucent adhesive tape. The light conditions were controlled for the test. Reflective light sources were removed, the illuminance was adjusted to $12-15 l \times$ by means of sun-blinds. The display of the tablet computer has been set to maximum brightness. For the augmentation only elevated parts of the graphics (see Appendix A) were illuminated by white light.

5.2.3 Design and Procedure. The user study used a within-subjects design. It consisted of five different tests, each available in three versions. One version was used for the briefing with the subject, the pretest, and the ability for the user to ask questions before the start of the actual test. The two other versions were used for the tests, to test our independent variable of visual augmentation, i.e., one of both versions utilized visual augmentation $\left(\mathrm{V}_{\mathrm{A}}\right)$, while the other version was not augmented $\left(\mathrm{V}_{\mathrm{N}}\right)$.

The tests were conducted with both test versions, but arrangements were made to prevent distortions by a possible learning effect between testing the augmented version against the nonaugmented version. For this purpose, the order of both versions was alternated for each user. In addition, we considered possible differences in the level of difficulty between both tested versions of each test. To compensate for this in the individual tests, the sequence of $\mathrm{V}_{\mathrm{A}}$ and $\mathrm{V}_{\mathrm{N}}$ was also alternated with each user (see Figure 7).

As can be seen from Figure 7, an introduction was first carried out with each subject. After the welcome she or he was asked about his general data (see Section 5.2.1: age, sex, visual acuity, and experience with tactile graphics). Next, it was explained that now five different tests are carried out, in which it is important to determine the correct result as quickly as possible. In addition to the sense of touch, the residual sight should always be used (for both $\mathrm{V}_{\mathrm{A}}$ and $\mathrm{V}_{\mathrm{N}}$ ). Then a tactile example was presented for each test, explaining the task to be solved. After probing the pattern, the subjects were asked to answer any questions before the actual test began. Additionally, the users were instructed to first determine the correct result, and then to report it. Re-checks and re-counts were not allowed. After completing the five tests, the purpose of the tests was explained and the user was asked for comments or suggestions for improvement.

For the exploration of tactile graphics, the accuracy and speed were identified as two important indicators. For this reason, our dependent variables were the number of errors (deviations of the detected variables from the actual graphics by means of formalized parameters) as well as the time required for recognition.

In this context, the following hypotheses are tested for each test:

$H_{0_{\text {Accuracy }}}$ : There is no significant difference in the number of errors between $V_{N}$ and $V_{A}$.

$H_{1_{\text {Accuracy }}}$ : With $\mathrm{V}_{\mathrm{A}}$, fewer errors are made than with $\mathrm{V}_{\mathrm{N}}$. 


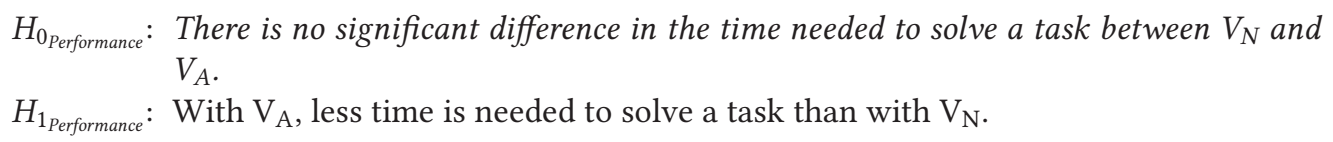

The following tests were carried out in this user study:

- Test 1

Simple geometric shapes are well known and can be described by simple characteristic numbers when they are recognized. For this purpose, we chose regular polygons, i.e., convex polygons and star polygons (see Appendix A), which had to be described by their number of corner vertices. These were arranged into four shapes in two rows, i.e., one row for the convex polygons and another row for the star polygons. The diameters of these shapes varied between 18 and $35 \mathrm{~mm}$. The subjects were instructed that these can have 3, 4, 5, 6, or 8 corners and that they should start at the top left and then report the numbers in the first row and then the second row. We recorded the time needed to solve this task and counted an error for each false number of vertices reported by the user. In $\mathrm{V}_{\mathrm{A}}$ the geometrical objects were illuminated.

- Test 2

The second test incorporated arrows (see Appendix A) to find out whether the visual enrichment can bring advantages in recognizing specific details in graphics (i.e., arrowheads). The total length of an arrow was $21 \mathrm{~mm}$, its arrow shaft had a thickness of $2.0 \mathrm{~mm}$ and the arrow tips had an angle of $30^{\circ}$. The subjects were instructed to report the directions (up, down, left, right) of eight different arrows from left to right. We recorded the time needed to solve this task and counted an error for each false direction reported by the user. In $V_{A}$ the arrows were illuminated.

- Test 3

This test should mainly determine whether the enrichment of negative tactile features is useful. For this purpose several circles were inscribed in raised rectangles, so that corresponding cavities were created (see Appendix A). The subjects had to determine the number of holes in this task, but they did not obtain any information how many holes are possible. They were advised that there may be three different hole diameters, but that these differences were not relevant. For the different hole diameters and the distances between them, the BANA guidelines [3] were taken into account. Three rectangles $(47 \times 67 \mathrm{~mm})$ were to be determined from left to right with hole diameters of 4,6 , and $9 \mathrm{~mm}$. We recorded the time needed to solve this task and counted the difference of the actual number of holes and the reported number of holes. In $\mathrm{V}_{\mathrm{A}}$ the holes were illuminated.

- Test 4

This test represented a line diagram with overlapping lines (see Appendix A). Beside this type of graphic itself, lines are often used to represent edges or borders in more complex graphic types such as illustrations. Tactile line styles are diverse and their combined use can lead to low readability in more complex graphics. Because of this, we aligned ourselves with the BANA guidelines, which makes suggestions about suitable combinations of line styles. The line width of each line was $2.0 \mathrm{~mm}$. The subjects were instructed that there are three different line styles (dotted, dashed, and continuous) that partially cross each other. Their task was to follow each individual line from its left end to its right end. In this test, the users could repeatedly ask if they were still on the right line with their fingers. We recorded the times needed to follow each of the lines. In this test, no errors were recorded. In $V_{A}$ the individual lines were illuminated. 
- Test 5

The last test represented a grouped bar graph with multiple tactile textures (see Appendix A). Similar to the lines, such textures are often used in more complex graphic types to represent surfaces. Since these textures are also diverse, we aligned ourselves with the BANA guidelines, which makes suggestions about suitable combinations of textures. The bars width was $7.5 \mathrm{~mm}$ and their height was variable. Three different textures (diagonally hatched, dotted, grid) were chosen. The participants were instructed to only count bars with a specific texture, whereas the height of the bars were not relevant. We recorded the time needed to solve this task and counted an error when there was a difference between the actual number of bars and the number of bars was reported by the user. In $V_{A}$ the individual bars were illuminated.

- Fixation of tactile overlays

This additional task had to be performed by each participant to find out if completely untrained users are able to attach the 3D printed tactile overlays to the tablet computer by two strips of adhesive tape. The tactile overlay was placed on the left side of the tablet computer and both strips of adhesive tape were stuck on the table. The participants were instructed to first place the overlay on the tablet computer and then to attach the adhesive tape on the left and right border of the overlay.

\subsubsection{Results.}

General observations. Neither in version $\mathrm{V}_{\mathrm{N}}$ nor $\mathrm{V}_{\mathrm{A}}$ a (linear) correlation $\left(\forall r: r^{2}<0.15\right)$ could be found between the visual acuity and the time needed to solve the tasks (see Appendix B). Even subjects with a very low visual acuity significantly benefitted from the visual augmentation. Subjects with a visual acuity of $\leq 2 \%$ achieved a $25 \%$ reduced rate of error with the visual speed increase and a speed gain of $108.94 \%$ compared to the respective non-augmented versions.

We performed a Mann-Whitney U-test to determine whether the distributions of the group of congenital visually impaired users differs from the group of late visually impaired users regarding the user performance (timings). For each individual test (both $V_{N}$ and $V_{A}$ ), we carried out the statistical test, but none of the results showed evidence that there was such a dependency (see Appendix C).

Individual test results. For each of the subjects, we received the number of errors and the time needed to solve the test for each of the five tests for both versions. For Tests 4 and 5, three timings were recorded. In addition, the wrong answers were documented. For the following statistical evaluation of the paired results, we used the nonparametric Wilcoxon Signed Rank Test [54].

- Test 1

The evaluation of Test 1 revealed a statistically significant faster problem solving by visually augmenting the tactile graphics. It resulted in $\mathrm{z}=-2.02, \mathrm{p}=0.022$, with a large effect size $(\mathrm{r}=0.5)$. The median time needed to solve this test decreased (see Figure 8) from $\mathrm{V}_{\mathrm{N}}(\mathrm{Md}=$ $48.34)$ to $\mathrm{V}_{\mathrm{A}}(\mathrm{Md}=40.94)$. There was no significant difference in the numbers of errors. This is mainly influenced by the fact that only few errors have been made, i.e., many users correctly identified each of the eight geometric shapes with $\left(\mathrm{N}_{\text {correct }}=10\right)$ and without visual augmentation $\left(\mathrm{N}_{\text {correct }}=8\right)$.

- Test 2

The evaluation revealed a statistically significant faster problem solving by visually augmenting the tactile graphics of Test 2. It resulted in $\mathrm{z}=-3.36, \mathrm{p}<0.001$, with a large effect size $(r=0.84)$. The median time needed to solve this test decreased (see Figure 9) from 


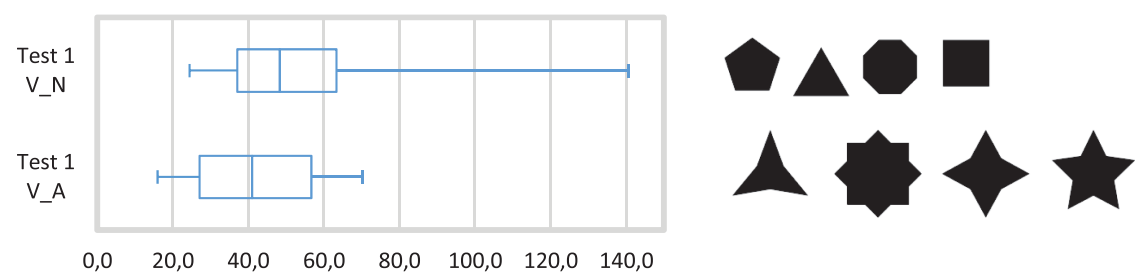

Fig. 8. Boxplot of user study results (timings in seconds) for Test 1 (left). Exemplary tactile graphics (right): black parts represent elevated areas that are conditionally augmented by white light.

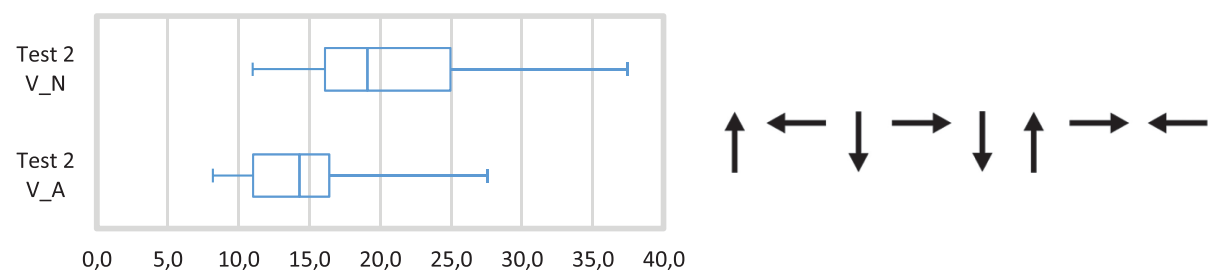

Fig. 9. Boxplot of user study results (timings in seconds) for Test 2 (left). Exemplary tactile graphics (right): black parts represent elevated areas that are conditionally augmented by white light.
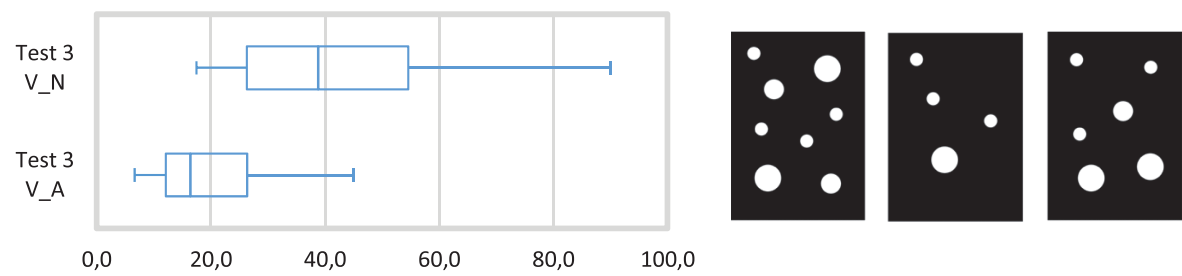

Fig. 10. Boxplot of user study results (timings in seconds) for Test 3 (left). Exemplary tactile graphics (right): black parts represent elevated areas that are conditionally augmented by white light.

without augmentation $(\mathrm{Md}=19.10)$ to with augmentation $(\mathrm{Md}=14.30)$. In this test, no significant difference in the number of errors could be determined. All participants except one completed the test without errors. This participant reported multiple errors in both $V_{N}(5)$ and $\mathrm{V}_{\mathrm{A}}(3)$.

- Test 3

In Test 3 the participants were significantly faster to find the rectangles' holes in $\mathrm{V}_{\mathrm{A}}$ compared to $\mathrm{V}_{\mathrm{N}}$. It resulted in $\mathrm{z}=-3.52, \mathrm{p}<0.001$, with a large effect size $(\mathrm{r}=0.88)$. The median time needed to solve this test decreased (see Figure 10) by more than $50 \%$ from $\mathrm{V}_{\mathrm{N}}(\mathrm{Md}=$ $38.83)$ to $\mathrm{V}_{\mathrm{A}}(\mathrm{Md}=16.44)$. The number of errors also significantly dropped $(\mathrm{z}=-2.29, \mathrm{p}=$ $0.011)$ from $\mathrm{V}_{\mathrm{N}}(\mathrm{Md}=1.50)$ to $\mathrm{V}_{\mathrm{A}}(\mathrm{Md}=0.00)$.

- Test 4

This test indicated that the recognition of individual lines can be significantly improved with visual augmentation $(z=-5.82, p<0.001$, with a large effect size $r=0.84)$. The median time needed to solve this test decreased (see Figure 11) from $\mathrm{V}_{\mathrm{N}}(\mathrm{Md}=12.11)$ to $\mathrm{V}_{\mathrm{A}}(\mathrm{Md}=5.74)$ by more than $50 \%$. Since in this test the participants were able to consult the experimenter to find out if they are touching the correct line, all the participants successfully completed this task. 


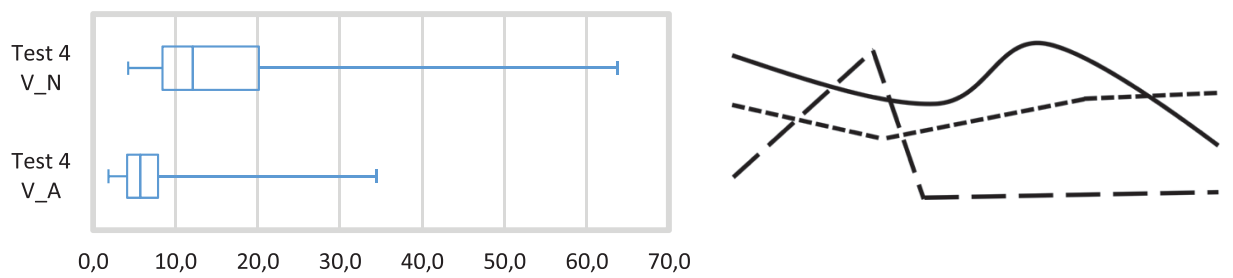

Fig. 11. Boxplot of user study results (timings in seconds) for Test 4 (left). Exemplary tactile graphics (right): black parts represent elevated areas that are conditionally augmented by white light.

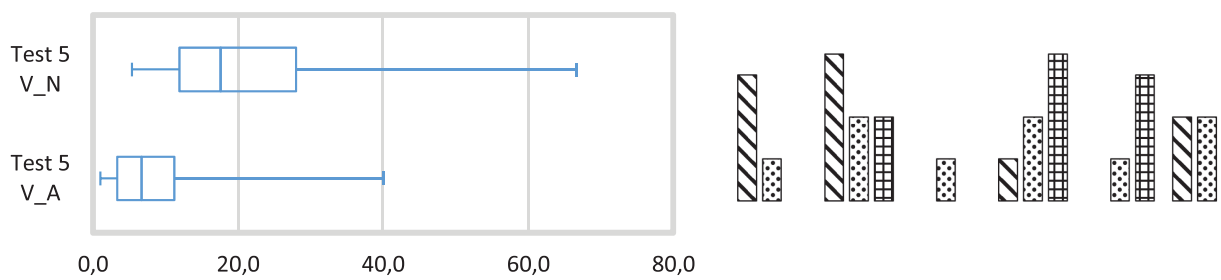

Fig. 12. Boxplot of user study results (timings in seconds) for Test 5 (left). Exemplary tactile graphics (right): black parts represent elevated areas that are conditionally augmented by white light.

- Test 5

The evaluation of Test 5 showed evidence that the recognition of individual textured elements can be significantly improved $(\mathrm{z}=-5.52, \mathrm{p}<.001$ with a large effect size $\mathrm{r}=.80)$ using visual augmentation. The median time needed to solve this test decreased (see Figure 12) from without augmentation $(\mathrm{Md}=17.61)$ to with augmentation $(\mathrm{Md}=6.72)$. Beside that the number of errors also significantly $(\mathrm{z}=-2.97, \mathrm{p}=.001)$ decreased from $\mathrm{V}_{\mathrm{A}}$ in relation to $\mathrm{V}_{\mathrm{A}}$.

- Fixation of tactile overlays

Each of the users successfully solved the task on the first attempt with an average time of $19.05 \pm 7.76 \mathrm{~s}$. Some of the users placed the tape diagonally across the edges of the overlay, others arranged the tape horizontally on the left and right edge of the overlay. However, in each case the tactile overlay was properly fixed on the tablet computer.

5.2.5 Discussion. The Hypothesis $H_{0_{\text {Performance }}}$ can be rejected for each of the tests, the time needed to solve the tasks significantly decreased from $\mathrm{V}_{\mathrm{N}}$ and $\mathrm{V}_{\mathrm{A}} . H_{0_{\text {Accuracy }}}$ can be rejected for Test 3 to 5 . For the Test 1 and 2 it cannot be rejected, which is probably related to the fact that in these test only few errors have been made. In total the average number of errors per user and test $\left(\mathrm{V}_{\mathrm{N}}\right.$ and $\left.\mathrm{V}_{\mathrm{A}}\right)$ was relatively low $(0.57)$, which could be related to a good legibility of the tactile materials.

In Test 3, three rectangles with either 4, 6, or 8 cavities were shown in this test for both versions $\mathrm{V}_{\mathrm{N}}$ and $\mathrm{V}_{\mathrm{A}}$. Their number had to be stated correctly, but the participants were not informed in advance which numbers are possible. In addition to the total time for the recognition of the numbers, it was also noted which numbers the subjects called the correct solutions. A further analysis was carried out using the Wilcoxon Signed Rank Test to determine whether the errors in the rectangles with 4,6 , or 8 cavities significantly decreased from $V_{N}$ to $V_{A}$. No significant difference was found in the rectangle with 4 cavities $(\mathrm{z}=-0.49, \mathrm{p}=0.312)$. In contrast, there was a significant decrease in the errors in the rectangles with 6 cavities $(\mathrm{z}=-1.83, \mathrm{p}=0.034)$ and 8 cavities $(\mathrm{z}=-2.50, \mathrm{p}=$ 0.006). Together with the results of the more complex graphs of Tests 4 and 5 , this could be an 
indication that the cognitive load (e.g., Reference [1]) of the subjects can be reduced by the additional visual support. A further study could examine further in detail the extent to which more complex graphics can be mediated by supporting the residual vision of visually impaired people.

The participants felt very positive about the additional visual augmentation. They mentioned in particular that the lighting can help to find relevant parts of tactile graphics. Even subjects with a very high visual impairment could benefit from this: "It sparkles somehow and helps to understand where one has to grope." The augmentation can be used to complement the audio-tactile graphics. They can be used in combination with textures (Test 5) to assign graphical elements with attributes or to show which elements belong together.

Some subjects found it very helpful that the background was dark and only important items in the foreground were bright-unlike many representations on screens and printed tactile graphics. One respondent remarked that "there were no duplications by the light," another that "no wavy fringes appear."

To our surprise, multiple subjects said that instead of a single color, several colors could be used to further differentiate the graphics: "You could try to choose color combinations for further distinction"; "Other colors could also be good." In addition to this suggestion, two subjects mentioned that a softer color than white might be more appropriate for highlighting. To avoid being blinded, red and orange could be more suitable. The fact that color combinations with respect to audiotactile graphics were considered important could be the basis for further studies. Thus, several graphical elements could be simultaneously augmented by different colors and the meaning of the colors explained by verbal reference. Possibly, this could offer advantages in understanding the spatial relation of several picture elements.

\section{LIMITATIONS}

This section combines the limitations of the general approach and the outcomes of both user studies as well as provides ideas for improvements and future research.

\subsection{Limitations of Experimental Application on Tactile Maps}

The application-driven user study showed the principal feasibility of the use case on tactile maps but it also indicated limitations and how the application could be improved in future.

Reliability of sensors. Besides the technical issues regarding the voice input, the magnetic sensor used for the compass functionality sometimes delivered skewed orientations. This is a common issue of electronic compasses built in mobile devices. A common strategy that is carried out to calibrate augmented reality applications is to wave the device once in an infinity-shaped manner before using the application. This is a simple but effective mean to calibrate the smartphone for the application presented in the feasibility study.

Map features included in map databases. The offline map material used by the Mapsforge framework is freely available on the internet. However, it is pre-processed to be stored efficiently in a compressed format, which also incidentally excludes some special tags for accessibility. By using existing tools, specialized map material for blind and visually impaired people could be generated from the OpenStreetMap database and provided to the community through the internet.

Optimal tactile representation. The effectiveness of (tactile) maps directly depends on their map design. The experimental application did not focus on tactile map design. Improving the map design could also improve the effectiveness of our approach.

A main challenge to tackle in the future is to identify how the tactile representation of (semi-) automatically created maps should be and how this can be technically achieved in general 
(including multiple zoom levels). Since a similar task has been solved for electronic visual maps decades ago, the authors believe that this can achieved for tactile maps as well. To realize this, the research results of user studies carried out to address these questions (e.g., References [4, 7, 58]) have to be transferred to formal rules for computing functional and usable maps. Likewise, for volumetric tactile material, there is an increasing number of publications (e.g., Reference [17]) that could help to optimize the usage of multiple elevation levels (such as sketched in Section 3.1) for tactile map design. This has to be done with current and upcoming technologies in mind. Multiple tactile reproduction technologies should be addressed by an integrative approach to reach most of the blind persons.

Redundant auditory and visual output. Auditory and visual output should ideally be redundant to support blind and visually impaired people in equal measure. In the experimental application there was still a bias towards visual augmentation. Especially, when the user prompted to highlight categories of map features (e.g., public transport), the application merely verbally reported how many of these map features were on the map, but not where they were located on the map. The individual map features were only visually highlighted.

As an improvement, future studies could address the verbalization of the position of individual map features and, ideally, a verbal description of their shape as a complement to the tactile exploration of map features by blind people.

\subsection{Limitations of Visual Augmentation}

The second user study addressed the effect of visual augmentation of tactile maps. It showed that even visual impaired people with less residual vision considerable benefitted from the augmentation for multiple kinds of graphics. This experiment has been carried out under laboratory conditions. Multiple potential limitations can be considered.

Lighting conditions. The visual augmentation of the translucent tactile graphics is dependent on lighting conditions of the environmental light. In bright areas (e.g., outdoors) this could impede the recognition of augmented map features. Similar issues could occur for speech interaction. However, this is also true for standard use of mobile devices for sighted users.

Partial opacity of filament. To the knowledge of the author, there is no existing 3D printer filament that is completely transparent. According to its specifications the filament used in the experiments only had $90 \%$ transparency ( $<1 \%$ haze). The fact that multiple printing layers have to be stacked on each other and the fabrication process itself (fused deposition modeling) are still reducing the transparency. To limit the opacity the layer height should be maximized, i.e., by printing as few as possible stacking layers.

Characteristics of displays. The characteristics of mobile devices' displays are manifold. In the user study of the effect of visual augmentation, we only used one specific display. We intentionally did not use a high-end device for the tests. However, parameters such as the resolution, brightness or color reproduction differ amongst the consumer devices and may influence the performance of users. We plan to carry out a comprehensive user study to assess different light conditions, 3D printing filaments, and display characteristics.

\subsection{General Limitations}

Some of the limitations are related to the general approach. Its applicability to specific usage scenarios depends on multiple parameters. 
Number of Codes. The use of capacitive markers with tactile graphics allows to identify the graphics immediately by touch. However, for this reason the marker has to be completely placed on the touch input, i.e., the number of distinguishable graphics directly depends on the touch input's size. Using the coding scheme proposed in Section 4 for smaller devices (e.g., 100mm width) such as smartphones only 55 codes can be used. However, the mobile usage (e.g., maps) is not designed to be used with a high number of tactile sheets that cover large areas. Instead, it can be used to provide orientation in the target area [9], such as a bus stop or a university campus or to recall map features. For tablet computers the original coding scheme provides 465 distinct codes for a touch input size of $200 \mathrm{~mm}$ width. If an application needs a higher number of distinguishable graphics, then the coding scheme can simply be extended by additional points touching the input (see codes $\mathrm{A}$ and $\mathrm{B}$ in Figure 2). Hence, adding an additional point $\mathrm{C}$ results in 3,276 codes, respectively, another point $\mathrm{D}$ in 12,650 codes.

Protective Cases. Smart phones are sometimes framed with protective cases. When bulky cases are used, this could prevent seamlessly placing a flat object on the touch surface. Despite this, we informally tested the use of tactile graphics with a low-cost smartphone the user studies focused on the use with a tablet computer. For the use of tactile graphics with smaller displays a dedicated comprehensive user study could reveal more practical issues.

Costs and Availability. As discussed in the introduction, costs are an important factor for many in the target group. Exemplary 3D printed tactile graphics of $10 \times 15 \mathrm{~cm}$ had an average weight of approximately $10 \mathrm{~g}$ and consisted of $\sim 90 \%$ transparent HDglass filament (750g: $\sim \$ 40)$ and $~ 10 \%$ conductive PLA (500g: \$48). On average, this means that the material costs of such a tactile graphics usually is below $\$ 0.60$. Even cheap 3D printers may be suitable for printing such graphics-we successfully tested prints with a dual-head 3D printer with a price point below $\$ 1,000$. Because there is a competitive market for 3D printers and filaments, it can be expected that this will continually drive quality and cost reduction of these mass products. The approach does not have demanding hardware requirements and can be used with affordable mobile devices. However, this could still limit the dissemination of applications using this approach, especially for people in the developing world.

Portability. In our usage scenario (see Section 4), we tested an application of our approach for tactile maps. As other types of graphics maps can be used for different purposes. Even though in our study one sheet only weighed $10 \mathrm{~g}$, when portability is an important characteristic for the intended usage scenario (e.g., in situ use of maps) the number of tactile sheets has to be limited. For the map use, we propose to use the tactile maps for supporting orientation in the target area [9]. For exploring large areas, we recommend using a larger map scale for the tactile graphics.

Limited layers. Using multiple elevation levels in tactile graphics could be used to organize information to support hierarchical scanning of tactile graphics. To allow touch (gestures) through the tactile sheets, the number of elevation levels is limited. Using off-the-shelf devices, the overall height is less than $1 \mathrm{~mm}$. However, reprogramming touch display controllers of consumer devices allows us to detect touch input from further distances [57], which could allow the exploration of spatial objects such as relief maps in future.

\section{CONCLUSION}

We presented a novel approach designed for both blind and visually impaired persons. It extends the concept of audio-tactile graphics by extending the visual modality. Particular graphical elements are visually augmented by illumination and groups of elements sharing content-related 
characteristics are emphasized. This allows visually impaired people to use their residual vision for the exploration of tactile graphics.

The following describes the individual contributions of this article about 3D printed Audiovisual Tactile Graphics. It presented a mobile solution for audio-tactile graphics, which can be used without any special equipment. The audio-tactile graphics work even with inexpensive standard smartphones and tablet computers.

We carried out a study of capacitive displays to obtain characteristics of proximity detection. By considering the requirements of 3D printers and the physiological characteristics of the human sense of touch, we developed a system that allows multiple elevation levels to in audio-tactile graphics. By assigning elevation levels to groups of graphical elements, this could be used to reduce tactile cluttering.

Our approach simplifies the combination of mobile devices with physical tactile graphics. By integrating conductive markers into the tactile graphics, the user is able to register graphics by a single touch or movement of the tactile graphics placed on a touch screen. It does not require the placement of the graphics in a specific way or aligned to a specific axis. Even completely oblique arrangements of the graphics can be used immediately. A sufficient fixation of the graphics is possible by simply attaching two strips of adhesive tape on the borders to the graphics. No extra attachment system is needed; the strips can be reused and temporarily stored on the mobile device's backside. Even untrained users are able to do this within few seconds.

The proposed solution of tactile graphics and integrated visual markers is printable with inexpensive off-the-shelf $3 \mathrm{D}$ printers in one turn without the need of post-processing. The material costs of these tactile graphics are comparable to prints on microcapsule paper.

Based on the developed generic concept on visually augmented audio-tactile graphics, we presented a case study for maps. The maps used for the case study can be semi-automatically generated by existing approaches (e.g., Reference [13]). We designed an interaction concept and adapted our approach to the needs for the audio-tactile exploration of maps. Finally, it was implemented prototypically and tested with a user study with visually impaired people. All the participants were able to couple the tactile graphics with a standard tablet computer within seconds and to interact with the 3D printed tactile maps. They visibly enjoyed the interaction with the visually augmented tactile maps.

To study the effect of visual augmentation, we conducted a comprehensive user study with another group of visually impaired people. We tested multiple types of graphics and obtained evidence that visual augmentation may offer clear advantages for the exploration of tactile graphics. Even participants with a residual vision of only $2 \%$ (WHO category 3/4 [8]) could solve the tasks with visual augmentation quicker and more accurately. Visually impaired people performed better in following linear tactile features, in counting elements, in exploring complex tactile graphics and in finding specific elements by visual augmentation. Furthermore, the results indicate that (i) the joint use of tactile sensation and visual feedback may reduce cognitive load, and (ii) (multicolored) augmentations could support the hierarchical scanning of tactile graphics. These results show interesting research questions to be targeted for future studies.

\section{APPENDIXES}

\section{A TEST OBJECTS OF USER STUDY ON VISUAL AUGMENTATION}

The white surfaces represent the basis of tactile graphics, while the black elements mean that the tactile graphics are raised by $0.3 \mathrm{~mm}$ at these positions. 
Test 1
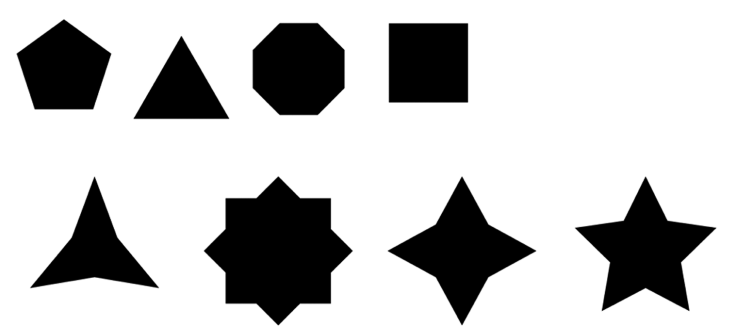

Test 2

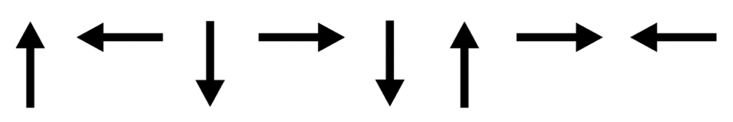

Test 3
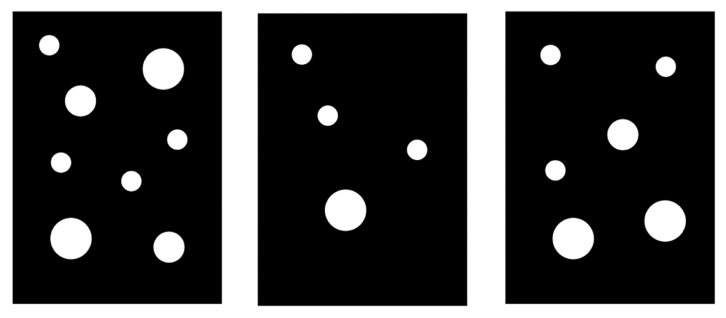

Test 4

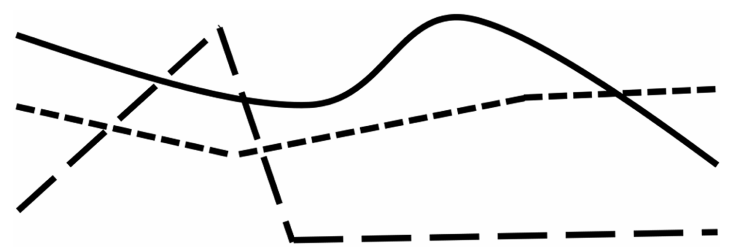

Test 5

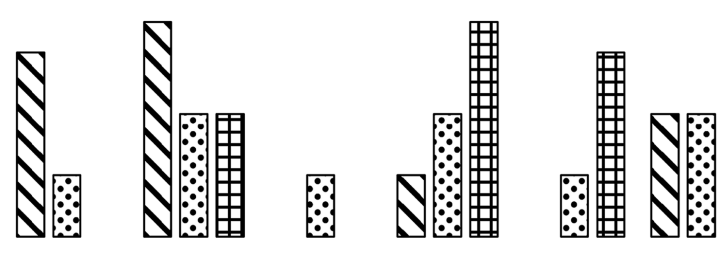

\section{B DETAILED SCATTER PLOTS}

This section shows scatter plots and the degree of correlation between acuity and problem solving time. Figures 13 to 17 illustrate that in none of the tests a dependency between both was variables was found. The elimination of the outlier (visual acuity=25\%) does not change this fact.

However, as described in Section 5.2.4, there is a considerable effect of visual augmentation (note that the Y scale of the scatter plots has been adapted for the sake of visualization). Even visually impaired people with a low level of residual vision considerably benefitted from the visual augmentation of tactile elements.

Figure 18 shows that the first test took more time for most of the users than the other tests. Tests 2 and 3 took almost the same time to solve the tasks. Figure 19 shows that the Tests 4 and 5 also took almost equal time to solve the tasks. Both figures indicate that users 2 and 14 performed slower than the remaining participants. However, there was no evident similarity regarding their age, acuity and type of visual impairment. Their acuity was nearly the average value (5\% and $10 \%$ ). 


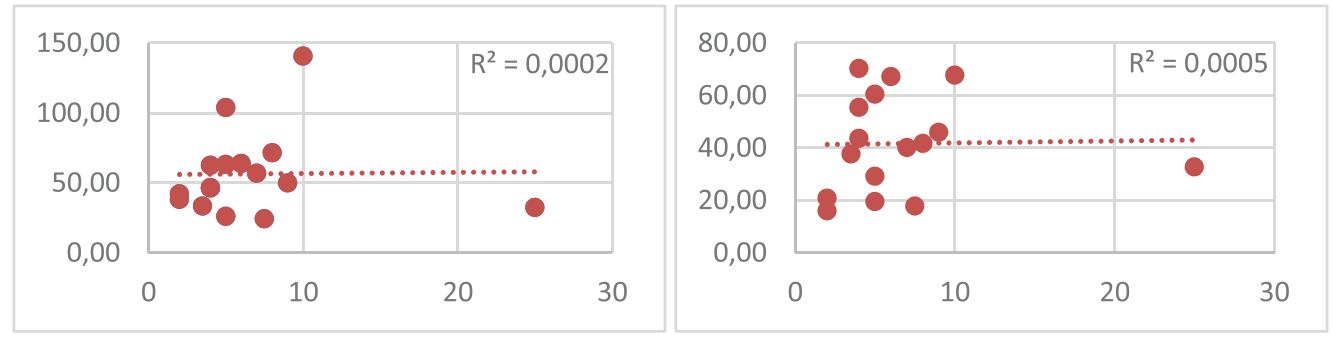

Fig. 13. Scatter plots for Test $1\left(\mathrm{X}\right.$, visual acuity; $\mathrm{Y}$, problem solving time). Without visual augmentation $\left(\mathrm{V}_{\mathrm{N}}\right)$ on the left with visual augmentation $\left(V_{A}\right)$ on the right.

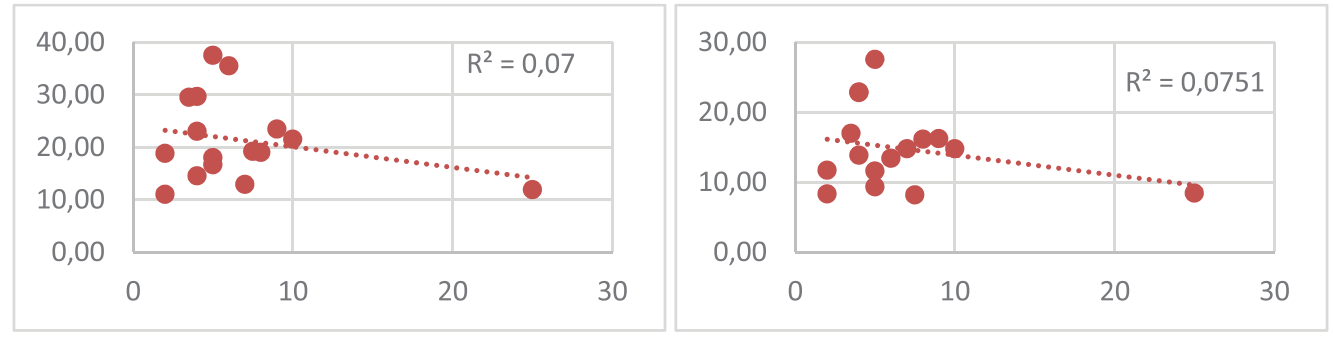

Fig. 14. Scatter plot for Test $2\left(\mathrm{X}\right.$, visual acuity; $\mathrm{Y}$, problem solving time). Without visual augmentation $\left(\mathrm{V}_{\mathrm{N}}\right)$ on the left with visual augmentation $\left(V_{A}\right)$ on the right.

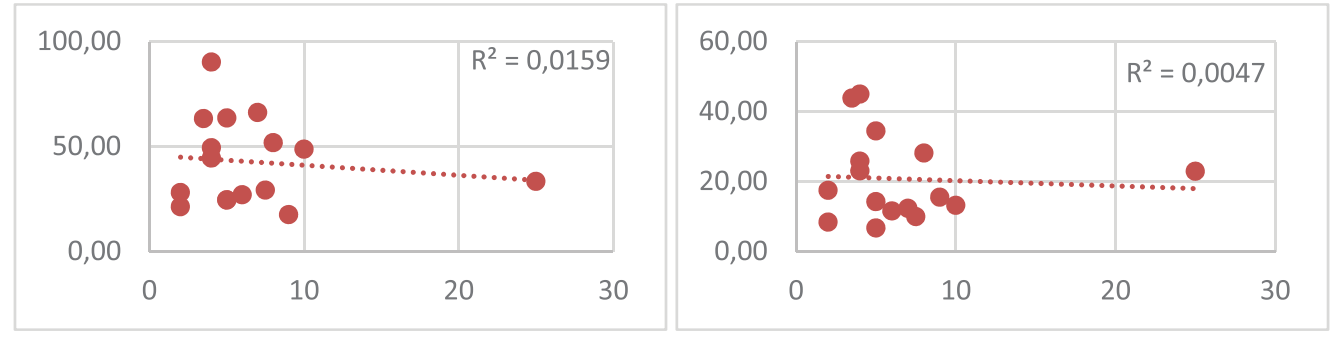

Fig. 15. Scatter plot for Test $3\left(\mathrm{X}\right.$, visual acuity; $\mathrm{Y}$, problem solving time). Without visual augmentation $\left(\mathrm{V}_{\mathrm{N}}\right)$ on the left with visual augmentation $\left(\mathrm{V}_{\mathrm{A}}\right)$ on the right.
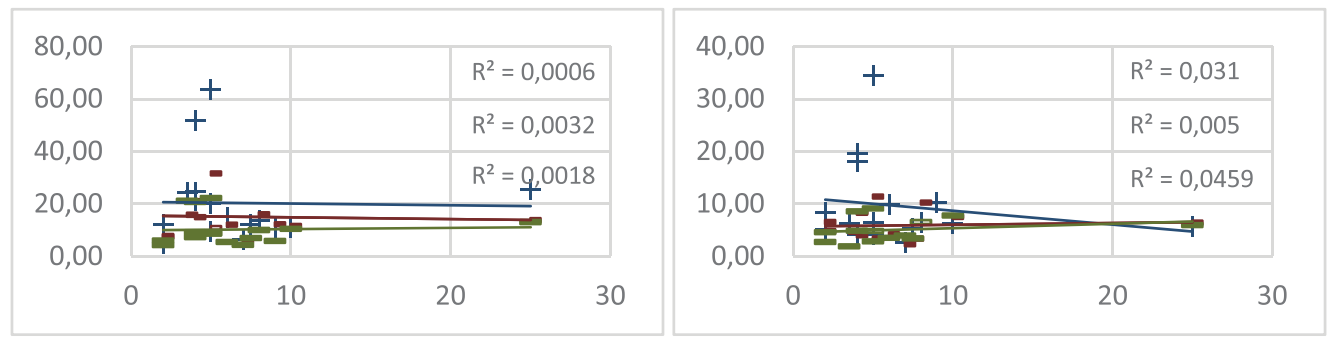

Fig. 16. Scatter plot for Test 4 (X, visual acuity; $\mathrm{Y}$, problem solving time). Without visual augmentation $\left(\mathrm{V}_{\mathrm{N}}\right)$ on the left with visual augmentation $\left(\mathrm{V}_{\mathrm{A}}\right)$ on the right side. Three passes were taken (1, blueish plus; 2 , greenish short dash; 3 , reddish long dash). 


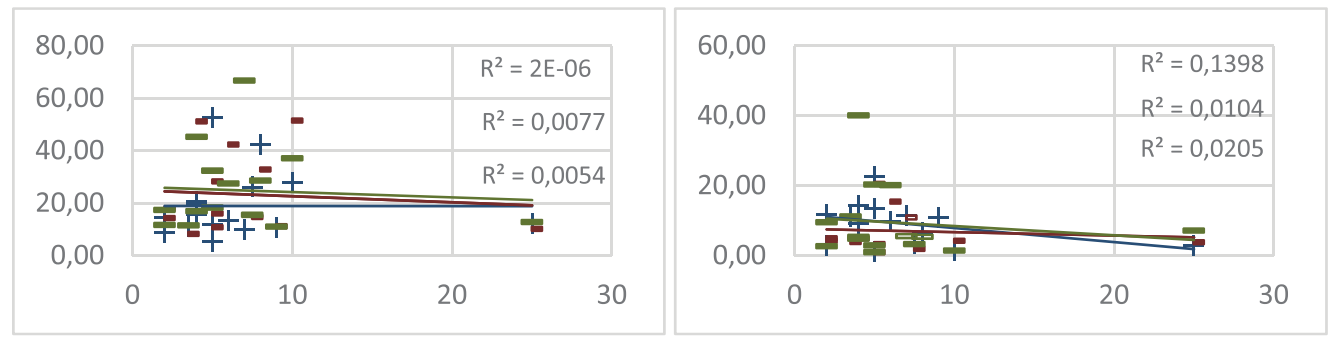

Fig. 17. Scatter plot for Test 5 (X, visual acuity; $\mathrm{Y}$, problem solving time). Without visual augmentation $\left(\mathrm{V}_{\mathrm{N}}\right)$ on the left with visual augmentation $\left(\mathrm{V}_{\mathrm{A}}\right)$ on the right side. Three passes were taken (1, blueish plus; 2 , greenish short dash; 3 , reddish long dash).

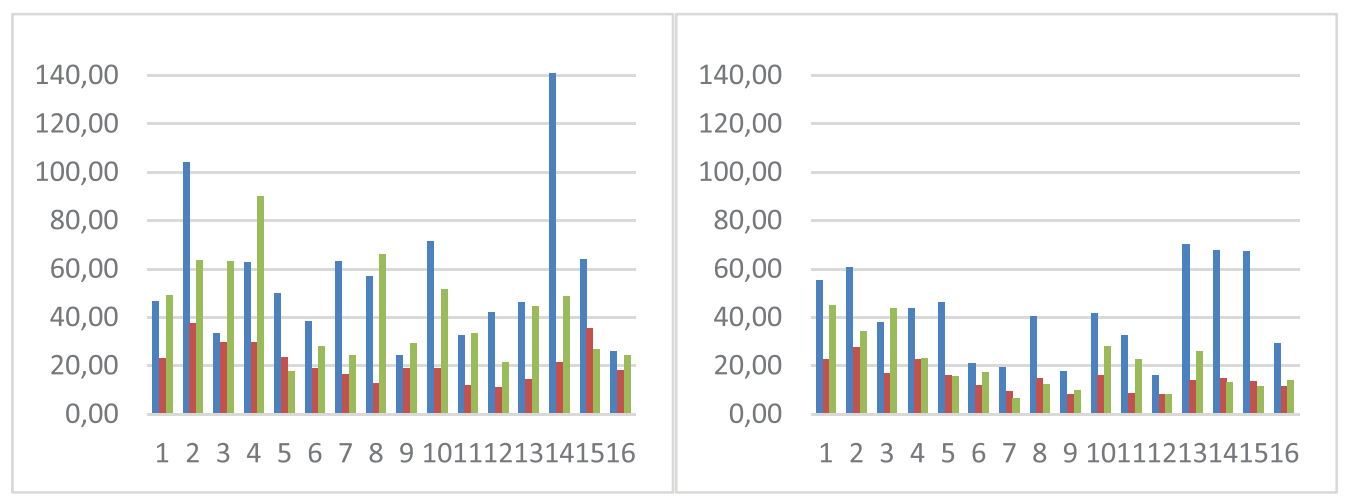

Fig. 18. Individual timings of users ( $x$, user ID; $y$, timings in seconds) across the tests (blue line, Test 1; red line, Test 2; green line, Test 3). Without visual augmentation $\left(\mathrm{V}_{\mathrm{N}}\right)$ on the left with visual augmentation (VA) on the right.

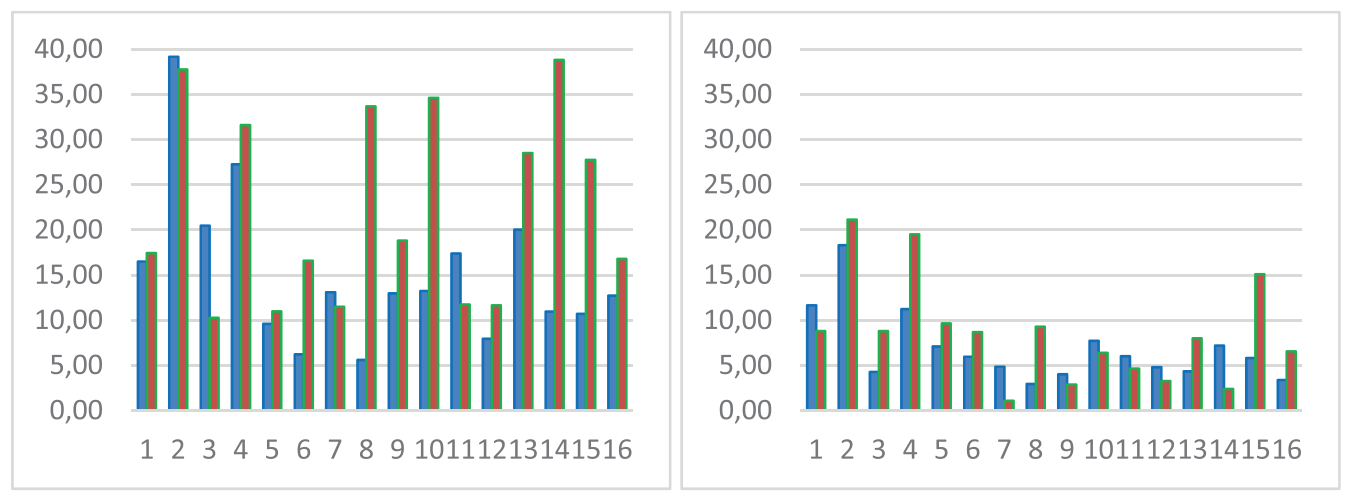

Fig. 19. Individual mean timings of users ( $x$, user ID; $y$, timings in $s$ ) across the tests (blue line, Test 4; green line, Test 5). Without visual augmentation (VN) on the left with visual augmentation (VA) on the right.

\section{PERFORMANCES OF CONGENITALLY VS. LATE VISUALLY IMPAIRED}

Table 2 shows the detailed results of the Mann-Whitney U-test described in Section 5.2.4. Group A $\left(N_{A}=10\right)$ represents late visually impaired people, whereas Group $B\left(N_{B}=6\right)$ represents 
congenitally visually impaired people. Its purpose was to test whether Group A performs better (regarding the problem solving time) than Group B (one-tailed, $\alpha=5 \%$ ).

Table 2. Detailed Test Results for Each Test

\begin{tabular}{|c|c|c|c|c|c|c|c|c|c|c|c|c|c|c|c|c|c|c|}
\hline & \multicolumn{2}{|c|}{ Test 1} & \multicolumn{2}{|c|}{ Test 2} & \multicolumn{2}{|c|}{ Test 3} & \multicolumn{6}{|c|}{ Test 4} & \multicolumn{6}{|c|}{ Test 5} \\
\hline & $\mathrm{V}_{\mathrm{N}}$ & $\mathrm{V}_{\mathrm{A}}$ & $\mathrm{V}_{\mathrm{N}}$ & $\mathrm{V}_{\mathrm{A}}$ & $\mathrm{V}_{\mathrm{N}}$ & $\overline{V_{N}}$ & $\mathrm{~V}_{\mathrm{N}}$ & $\mathrm{V}_{\mathrm{N}}$ & $\mathrm{V}_{\mathrm{N}}$ & $\overline{V_{A}}$ & $\overline{V_{A}}$ & $\mathrm{~V}_{\mathrm{A}}$ & $\mathrm{V}_{\mathrm{N}}$ & $\mathrm{V}_{\mathrm{N}}$ & $\overline{V_{N}}$ & $\mathrm{~V}_{\mathrm{A}}$ & $\mathrm{V}_{\mathrm{A}}$ & $\mathrm{V}_{\mathrm{A}}$ \\
\hline $\mathrm{n}_{1}$ & 10 & 10 & 10 & 10 & 10 & 10 & 10 & 10 & \begin{tabular}{|l|}
10 \\
\end{tabular} & 10 & 10 & 10 & 10 & 10 & 10 & 10 & 10 & 10 \\
\hline $\mathrm{n}_{2}$ & 6 & 6 & 6 & 6 & 6 & 6 & 6 & 6 & 6 & 6 & 6 & 6 & 6 & 6 & 6 & 6 & 6 & 6 \\
\hline$\overline{\mathrm{U}}$ & 17 & 17 & 26 & 21 & 17 & 30 & 25 & 27 & \begin{tabular}{|l|}
28 \\
\end{tabular} & 27 & 28 & 18 & 30 & 17 & 12 & 29 & 28 & 20 \\
\hline $\mathrm{U}_{\text {crit }}$ & 10 & 10 & 10 & 10 & 10 & 10 & 10 & 10 & 10 & 10 & 10 & 10 & 10 & 10 & 10 & 10 & 10 & 10 \\
\hline
\end{tabular}

None of the test statistics $\mathrm{U}$ was lower than $\mathrm{U}_{\text {crit. }}$.

\section{ACKNOWLEDGMENTS}

Thanks to the Educational Center for Blind and Visually Impaired People (BBS Nürnberg) and the Vocational Training Centre for the Blind and Visually Impaired (BFW Würzburg) as well as everyone involved in both user studies.

\section{REFERENCES}

[1] Catherine M. Baker, Lauren R. Milne, Ryan Drapeau, Jeffrey Scofield, Cynthia L. Bennett, and Richard E. Ladner. 2016. Tactile graphics with a voice. ACM Trans. Access. Comput. 8, 1, 1-22. Retrieved from https://doi.org/10.1145/2854005.

[2] Blender Foundation. blender.org-Home of the Blender project-Free and open 3D creation software. Retrieved from http://www.blender.org/.

[3] Braille Authority of North America. 2017. Braille Authority of North America: Guidelines and standards for tactile graphics. Retrieved from http://www.brailleauthority.org/tg/web-manual/index.html.

[4] Anke M. Brock. 2013. Interactive maps for visually impaired people: Design, usability, and spatial cognition. Université Toulouse 3 Paul Sabatier. Retrieved from http://tel.archives-ouvertes.fr/tel-00934643/.

[5] Anke M. Brock, Philippe Truillet, Bernard Oriola, Delphine Picard, and Christophe Jouffrais. 2015. Interactivity improves usability of geographic maps for visually impaired people. Hum.-Comput. Interact. 30, 2, 156-194.

[6] Craig Brown and Amy Hurst. 2012. VizTouch: Automatically generated tactile visualizations of coordinate spaces. In Proceedings of the 6th International Conference on Tangible, Embedded and Embodied Interaction, 131-138. Retrieved from http://dl.acm.org/citation.cfm?id=2148160.

[7] Emeline Brulé, Gilles Bailly, Anke M. Brock, Frédéric Valentin, Grégoire Denis, and Christophe Jouffrais. 2016. MapSense: Multi-sensory interactive maps for children living with visual impairments. In Proceedings of the ACM Conference on Computer-Human Interaction (CHI'16).

[8] Andrew Crossan and Stephen Brewster. 2008. Multimodal trajectory playback for teaching shape information and trajectories to visually impaired computer users. ACM Trans. Access. Comput. 1, 2, 12.

[9] M. Espinosa, Simon Ungar, Esperanza Ochaía, Mark Blades, and Christopher Spencer. 1998. Comparing methods for introducing blind and visually impaired people to unfamiliar urban environments. f. Environ. Psychol. 18, 3: 277-287.

[10] Tyler J. Ferro and Dianne T. V. Pawluk. 2013. Automatic image conversion to tactile graphic. In Proceedings of the 15th International ACM SIGACCESS Conference on Computers and Accessibility. 39.

[11] Timo Götzelmann. 2016. LucentMaps: 3D printed audiovisual tactile maps for blind and visually impaired people. In Proceedings of 18th International ACM SIGACCESS Conference on Computers and Accessibility (ASSETS'16). 81-90. Retrieved from https://doi.org/10.1145/2982142.2982163.

[12] Timo Götzelmann. 2016. CapMaps. In Proceedings of the 15th International Conference on Computers Helping People with Special Needs (ICCHP'16). Springer, 146-152.

[13] Timo Götzelmann and Laura Eichler. 2016. BlindWeb maps-An interactive web service for the selection and generation of personalized audio-tactile maps. In Computers Helping People with Special Needs, Klaus Miesenberger, Christian Bühler and Petr Penaz (eds.). Springer International Publishing, Cham, 139-145.

[14] Timo Götzelmann and Aleksander Pavkovic. 2014. Towards automatically generated tactile detail maps by 3D printers for blind persons. In Proceedings of the 14th International Conference on Computers Helping People with Special Needs (ICCHP'14). 1-7. 
[15] Timo Götzelmann and Daniel Schneider. 2016. CapCodes: Capacitive 3D printable identification and on-screen tracking for tangible interaction. In Proceedings of 9th Nordic Conference on Human-Computer Interaction (NordiCHI'16), Article No. 32. Retrieved from https://doi.org/10.1145/2971485.2971518.

[16] Timo Götzelmann and Klaus Winkler. 2015. SmartTactMaps: A smartphone-based approach to support blind persons in exploring tactile maps. In Proceedings of the 8th International Conference on Pervasive Technologies Related to Assistive Environments (PETRAE'15). 1-8. Retrieved from https://doi.org/10.1145/2769493.2769497.

[17] Jaume Gual, Marina Puyuelo, and Joaquim Lloveras. 2015. The effect of volumetric (3D) tactile symbols within inclusive tactile maps. Appl. Ergonom. 48, 1-10.

[18] Ronald A. L. Hinton. 1993. Tactile and audio-tactile images as vehicles for learning. In Proceedings of the INSERMSETAA Conference 228 (1993), 169-179.

[19] David Huggins-Daines, Mohit Kumar, Arthur Chan, Alan W. Black, Mosur Ravishankar, and Alex I. Rudnicky. 2006. Pocketsphinx: A free, real-time continuous speech recognition system for hand-held devices. In Proceedings of the IEEE International Conference on Acoustics, Speech and Signal Processing. 185-188.

[20] Rubén Iglesias, Sara Casado, T. Gutierrez, J. I. Barbero, C. A. Avizzano, S. Marcheschi, and M. Bergamasco. 2004. Computer graphics access for blind people through a haptic and audio virtual environment. In Proceedings of the $3 r d$ IEEE International Workshop on Haptic, Audio and Visual Environments and Their Applications (HAVE'04). 13-18.

[21] R. Dan Jacobson. 1998. Navigating maps with little or no sight: An audio-tactile approach. In Proceedings of the Workshop on Content Visualization and Intermedia Representations (CVIR'98). 95-102.

[22] Chandrika Jayant, Matt Renzelmann, Dana Wen, Satria Krisnandi, Richard Ladner, and Dan Comden. 2007. Automated tactile graphics translation: In the field. In Proceedings of the 9th International ACM SIGACCESS Conference on Computers and Accessibility. 75-82.

[23] Sandra Jehoel, Snir Dinar, Don McCallum, Jonathan Rowell, and Simon Ungar. 2005. A scientific approach to tactile map design: Minimum elevation of tactile map symbols. In Proceedings of 22nd International Cartographic Conference a Coruña (CD’05).

[24] Sandra Jehoel, Don McCallum, Jonathan Rowell, and Simon Ungar. 2006. An empirical approach on the design of tactile maps and diagrams: The cognitive tactualization approach. Brit. F. Visual Impair.t 24, 2, 67-75.

[25] Shaun K. Kane, Brian Frey, and Jacob O. Wobbrock. 2013. Access lens: A gesture-based screen reader for real-world documents. In Proceedings of the SIGCHI Conference on Human Factors in Computing Systems. 347-350.

[26] Shaun K. Kane, Meredith Ringel Morris, and Jacob O. Wobbrock. 2013. Touchplates: Low-cost tactile overlays for visually impaired touch screen users. In Proceedings of the 15th International ACM SIGACCESS Conference on Computers and Accessibility. 22.

[27] Jeeeun Kim and Tom Yeh. 2015. Toward 3D-printed movable tactile pictures for children with visual impairments. In Proceedings of the 33rd Annual ACM Conference on Human Factors in Computing Systems. 2815-2824.

[28] Marius Kintel and Clifford Wolf. OpenSCAD-The Programmers Solid 3D CAD Modeller. Retrieved from http://www. openscad.org/.

[29] Sven Kratz, Tilo Westermann, Michael Rohs, and Georg Essl. 2011. CapWidgets: Tangible widgets versus multi-touch controls on mobile devices. In Proceedings of the ACM Conference on Computer-Human Interaction (CHI'11). 1351-1356.

[30] Stephen E. Krufka and Kenneth E. Barner. 2005. Automatic production of tactile graphics from scalable vector graphics. In Proceedings of the 7th International ACM SIGACCESS Conference on Computers and Accessibility. 166-172.

[31] Robert H. LaMotte and Mandayam A. Srinivasan. 1991. Surface microgeometry: Tactile perception and neural encoding. In Information Processing in the Somatosensory System. Macmillan Education, UK, 49-58.

[32] Steven Landau and Lesley Wells. 2003. Merging tactile sensory input and audio data by means of the talking tactile tablet. In Proceedings of the European Conference on Haptics (EuroHaptics'03). 414-418.

[33] Nan Li, Zheshen Wang, Jesus Yuriar, and Baoxin Li. 2011. Tactileface: A system for enabling access to face photos by visually-impaired people. In Proceedings of the 16th International Conference on Intelligent User Interfaces. 445-446.

[34] M. Rotard and T. Ertl. 2006. Tactile 3D-Graphics for blind people. In Proceedings of the Workshop on Accessible Media. Retrieved from http://cumbia.informatik.uni-stuttgart.de/ger/research/pub/pub2006/Accessible-Media-2006-Rotard. pdf.

[35] David McGookin, Stephen Brewster, and WeiWei Jiang. 2008. Investigating touchscreen accessibility for people with visual impairments. In Proceedings of the 5th Nordic Conference on Human-computer Interaction: Building Bridges. 298-307.

[36] Joshua. A. Miele, Steven Landau, and Deborah Gilden. 2006. Talking TMAP: Automated generation of audio-tactile maps using smith-kettlewell's TMAP software. Brit. f. Visual Impair. 24, 2: 93-100. Retrieved from https://doi.org/10. $1177 / 0264619606064436$.

[37] Don Parkes. 1988. NOMAD: An audio-tactile tool for the acquisition, use and management of spatially distributed information by partially sighted and blind persons. In Proceedings of 2nd International Conference on Maps and Graphics for Visually Disabled People. 24-29. 
[38] Grégory Petit, Aude Dufresne, Vincent Levesque, Vincent Hayward, and Nicole Trudeau. 2008. Refreshable tactile graphics applied to schoolbook illustrations for students with visual impairment. In Proceedings of the 10th International ACM SIGACCESS Conference on Computers and Accessibility. 89-96.

[39] Benjamin Poppinga, Charlotte Magnusson, Martin Pielot, and Kirsten Rassmus-Gröhn. 2011. TouchOver map: Audiotactile exploration of interactive maps. In Proceedings of the 13th International Conference on Human Computer Interaction with Mobile Devices and Services. 545-550. Retrieved from http://dl.acm.org/citation.cfm?id=2037458.

[40] Bernhard Schmitz and Thomas Ertl. 2012. Interactively displaying maps on a tactile graphics display. In Proceedings of the Conference on Spatial Knowledge Acquisition with Limited Information Displays (SKALID'12). 13.

[41] Caterina Senette, Maria Claudia Buzzi, Marina Buzzi, Barbara Leporini, and Loredana Martusciello. 2013. Enriching graphic maps to enable multimodal interaction by blind people. In Universal Access in Human-Computer Interaction. Design Methods, Tools, and Interaction Techniques for eInclusion. Springer, 576-583. Retrieved from http://link.springer. com/chapter/10.1007/978-3-642-39188-0_62.

[42] Lei Shi, Idan Zelzer, Catherine Feng, and Shiri Azenkot. 2016. Tickers and talker: An accessible labeling toolkit for 3D printed models. 4896-4907. Retrieved from https://doi.org/10.1145/2858036.2858507.

[43] Jing Su, Alyssa Rosenzweig, Ashvin Goel, Eyal de Lara, and Khai N. Truong. 2010. Timbremap: Enabling the visuallyimpaired to use maps on touch-enabled devices. In Proceedings of the 12th International Conference on Human Computer Interaction with Mobile Devices and Services. 17-26.

[44] Saiganesh Swaminathan, Thijs Roumen, Robert Kovacs, David Stangl, Stefanie Mueller, and Patrick Baudisch. 2016. Linespace: A sensemaking platform for the blind. In Proceedings of the 2016 CHI Conference on Human Factors in Computing Systems. 2175-2185. Retrieved from https://doi.org/10.1145/2858036.2858245.

[45] A. F. Tatham. 1991. The design of tactile maps: Theoretical and practical considerations. Proceedings of the International Cartographic Association: Mapping the Nations. 157-166.

[46] Simon Voelker, Kosuke Nakajima, Christian Thoresen, Yuichi Itoh, Kjell Ivar Øvergård, and Jan Borchers. 2013. PUCs: Detecting transparent, passive untouched capacitive widgets on unmodified multi-touch displays. In Proceedings of the ACM International Conference on Interactive Tabletops and Surfaces. 101-104.

[47] Vít Voženílek, Magdaléna Kozáková, Zuzana Št'ávová, Libuše Ludíková, Veronika Rǔžičková, and Dita Finková. 2009. 3D Printing technology in tactile maps compiling. In Proceedings of 24th International Cartographic Conference, International Cartographic Association.

[48] Steven Wall and Stephen Brewster. 2006. Feeling what you hear: Tactile feedback for navigation of audio graphs. In Proceedings of the SIGCHI Conference on Human Factors in Computing Systems. 1123-1132.

[49] Zheshen Wang, Baoxin Li, Terri Hedgpeth, and Teresa Haven. 2009. Instant tactile-audio map: enabling access to digital maps for people with visual impairment. In Proceedings of the 11th International ACM SIGACCESS Conference on Computers \& Accessibility. 43-50. Retrieved from http://dl.acm.org/citation.cfm?id=1639652.

[50] Tetsuya Watanabe and Susumu Oouchi. 2003. A study on legible braille pattern on capsule paper: Diameters of braille dots and their interspaces on the original ink-printed paper. Bull. Natl. Inst. Special Edu. 30, 1-8.

[51] Thomas P. Way and Kenneth E. Barner. 1997. Automatic visual to tactile translation. II. evaluation of the TACTile image creation system. IEEE Trans. Rehabil. Engineer. 5, 1, 95-105.

[52] Thomas P. Way and Kenneth E. Barner. 1997. Automatic visual to tactile translation. i. Human factors, access methods, and image manipulation. IEEE Trans. Rehabil. Engineer. 5, 1, 81-94.

[53] Alexander Wiethoff, Hanna Schneider, Michael Rohs, Andreas Butz, and Saul Greenberg. 2012. Sketch-a-TUI: Low cost prototyping of tangible interactions using cardboard and conductive ink. In Proceedings of the Conference on Tangible, Embedded, and Embodied Interaction (TEI'12). 309-312.

[54] Frank Wilcoxon. 1945. Individual comparisons by ranking methods. Biometr. Bull. 1, 6, 80-83.

[55] World Health Organization. 2016. Visual impairment and blindness. Retrieved from http://www.who.int/ mediacentre/factsheets/fs282/en/.

[56] World Health Organization. 2017. Change the definition of blindness. Retrieved from http://www.who.int/blindness/ Change\%20the\%20Definition\%20of\%20Blindness.pdf?ua=1.

[57] Robert Xiao, Julia Schwarz, and Chris Harrison. 2015. Estimating 3D finger angle on commodity touchscreens. 47-50. Retrieved from https://doi.org/10.1145/2817721.2817737.

[58] Limin Zeng, Mei Miao, and Gerhard Weber. 2015. Interactive audio-haptic map explorer on a tactile display. Interact. Comput. 27, 4, 413-429. Retrieved from https://doi.org/10.1093/iwc/iwu006.

[59] Limin Zeng and Gerhard Weber. 2011. Accessible maps for the visually impaired. In Proceedings of the IFIP INTERACT Workshop on Accessible Design in the Digital World. 54-60.

[60] Limin Zeng and Gerhard Weber. 2012. ATMap: Annotated tactile maps for the visually impaired. In Cognitive Behavioural Systems. Springer, 290-298.

Received April 2017; revised February 2018; accepted February 2018 